\title{
The Therapeutic Properties of Plants Used Traditionally to Treat Gastrointestinal Disorders on Groote Eylandt, Australia
}

\author{
Cécile Mazerand $^{1,2}$ and Ian Edwin Cock $\mathbb{D i D}^{1,3}$ \\ ${ }^{1}$ School of Environment and Science, Griffith University, Brisbane 4111, Australia \\ ${ }^{2}$ School of Biology, Ecole de Biologie Industrielle (EBI), Cergy, France \\ ${ }^{3}$ Environmental Futures Research Institute, Griffith University, Brisbane, Australia \\ Correspondence should be addressed to Ian Edwin Cock; i.cock@griffith.edu.au
}

Received 21 May 2020; Revised 10 September 2020; Accepted 28 October 2020; Published 10 November 2020

Academic Editor: Carlos H. G. Martins

Copyright ( 92020 Cécile Mazerand and Ian Edwin Cock. This is an open access article distributed under the Creative Commons Attribution License, which permits unrestricted use, distribution, and reproduction in any medium, provided the original work is properly cited.

\begin{abstract}
The First Australians had well-developed healing systems. Groote Eylandt inhabitants used a variety of plant species to treat diarrhoea and other gastrointestinal illnesses. This study was undertaken to test, identify, and evaluate traditional medicines to treat these conditions against gastrointestinal bacterial, protozoal, and viral pathogens, as well as against cancer cell proliferation. Six plant species (Buchanania obovata Engl., Casuarina equisetifolia L., Eucalyptus tetrodonta F. Muell., Planchonia careya (F. Muell.) R. Knuth, Terminalia carpentariae C. T. White, and Vigna vexillata (L.) A. Rich.) were selected from a survey of a panel of elders from the Warnindhilyagwa tribe and compared with the published literature. Decoctions prepared according to traditional methods were screened for growth inhibitory activity of a panel of diarrhoea-causing bacterial pathogens by disc diffusion and liquid dilution MIC assays. Inhibitory activity against the gastrointestinal protozoal parasite Giardia duodenalis and antiproliferative activity against human colorectal (Caco2) and cervical (HeLa) cancer cell lines were evaluated using MTS-based colorimetric cell proliferation assays. Preliminary antiviral screening was accomplished using an MS2 bacteriophage plaque reduction assay. Toxicity was evaluated using Artemia franciscana nauplii mortality and HDF cell viability bioassays. All traditional medicines tested inhibited bacterial growth, often with MIC values substantially $<1000 \mu \mathrm{g} / \mathrm{mL}$. T. carpentariae was particularly noteworthy, with MIC values of $230-350 \mu \mathrm{g} / \mathrm{mL}$ against Citrobacter freundii, Salmonella newport, Shigella sonnei, Staphylococcus aureus, and Staphylococcus epidermidis. This species also had MICs $450-950 \mu \mathrm{g} / \mathrm{mL}$ against all other bacterial pathogens. B. obovata Engl. and E. tetrodonta were also good inhibitors of bacterial growth, albeit with substantially higher MIC values than determined for T. carpentariae. The T. carpentariae decoction was also the best inhibitor of MS2 phage replication $\left(\mathrm{IC}_{50}=427 \mu \mathrm{g} / \mathrm{mL}\right)$ and $\mathrm{Caco} 2$ and HeLa proliferation $\left(\mathrm{IC}_{50}\right.$ values of 885 and $85 \mu \mathrm{g} / \mathrm{mL}$, respectively). None of the extracts were particularly strong inhibitors of Giardia duodenalis growth. All decoctions were nontoxic in the Artemia nauplii and HDF cell viability bioassays, indicating their suitability for therapeutic use.
\end{abstract}

\section{Introduction}

The World Health Organization (WHO) has estimated that nearly nine million children under the age of five die every year as a result of diarrhoea [1]. Despite that report being more than a decade old, very little has changed in the interim, and diarrhoea remains the leading killer of children globally, accounting for approximately $9 \%$ of all deaths among children under the age of five [2]. This translates into more than 1400 young children dying each day, or about
530,000 children a year. To exacerbate this problem, many bacteria have developed resistance to conventional antibiotics, rendering them of little use against some diarrhoeacausing pathogens [3]. There is an urgent need to develop new treatment options to combat these diseases through the development of novel drugs.

Plants have long been used in traditional healing systems to treat diarrhoea. These traditional medicines may be given as single-component therapies or they may be prescribed in combination to target the multiple negative effects of 
diarrhoea (loose stools, cramps, loss of electrolytes, and possible fever). The activity of several of the herbal preparations used traditionally to treat diarrhoea and other gastrointestinal diseases has already been validated by rigorous scientific evaluation. This is particularly true for plant medicines used in traditional Indian healing systems (including Ayurveda, Siddha, and Unani) [4-6] and in traditional Chinese medicine (TCM) [7, 8]. In contrast, the potential of many Australian medicinal plants in alleviating the symptoms of diarrhoea and inhibiting the pathogenic causes has been relatively neglected.

Despite being one of the oldest continuous civilisations globally, far less information is available describing the ethnobotany of the First Australians than for many other ethnic groups globally. The First Australians had a good understanding of the medicinal properties of Australian plants and had used them successfully for at least 50,000 years to treat a variety of illnesses. However, little of this information has been recorded as the First Australians did not have a written language. Instead, traditional knowledge was passed between generations orally, and most ethnobotanical texts have collated data from the perspective of European settlers. As a result, substantial ethnobotanical information has been irretrievably lost. Furthermore, many of the seminal texts that summarise Aboriginal plant usage are old and difficult to access, taxonomic classifications are outdated and often dubious, and the information is incomplete. Compounding the problem, the First Australians consist of diverse groups, with individual communities having their own language, culture, customs, and belief systems, as well as their own traditional knowledge. Members of one language group were generally unable to communicate effectively with other groups. Within the language groupings, the First Australians are further divided into local groupings, which often had regional dialects, further complicating communication. Furthermore, many of the First Australian groups lived in isolation from other groups. As such, exchange of knowledge between groups was often not viable. Additionally, Torres Strait Islanders have distinct heritage from the Aborigines, with substantially different customs and traditional knowledge. Thus, plants used traditionally by one group of First Australians may not have been used for the same purposes by other groups. Despite this relative dearth of rigorous ethnobotanical studies on Australian flora, several studies have identified plant species with noteworthy antimicrobial activity. In particular, the ethnobotany of the inhabitants of Groote Eylandt has been relatively well recorded, allowing for selection of relevant species to treat microbial pathogens [9].

Bacterial pathogens are major causes of diarrhoea, and several studies have already screened Australian medicinal plants for antidiarrheal properties. Palombo and Semple investigated the growth inhibitory properties of extracts prepared from 39 Australian plant species against a panel of bacterial pathogens, including several diarrhoea-causing species [10]. That study reported that several species had noteworthy inhibitory activity and highlighted Eremophila duttonii F. Muell. as having the greatest activity of the species tested. However, that study screened the plant extracts at a single dose (concentration not specified), and MIC values were not reported, making a comparison with other studies impossible. Furthermore, the Palombo and Semple study tested ethanolic extracts instead of the decoctions that were used by the First Australians. Therefore, whilst that study was interesting and highlighted several plants worthy of further evaluation, it did not validate the traditional use of those species to treat diarrhoea. Another study screened extracts prepared from 109 Australian plants for the ability to inhibit the growth of Campylobacter jejuni, which is one of the most common causes of acute enteritis in humans, and reported potent activity for many species [11]. That study reported MIC values below $1000 \mu \mathrm{g} / \mathrm{mL}$ for all species against two $C$. jejuni strains and similar potency against several other diarrhoea-causing bacteria. Several Eucalyptus spp. were amongst the most potent species tested in that study. Several recent studies have also reported good inhibitory activity for several high-antioxidant Australian plant species against multiple bacterial species, including diarrhoea-causing pathogens [12-14]. A recent study also tested Terminalia ferdinandiana Excell. extracts against multiresistant ESBL Escherichia coli and methicillin-resistant Staphylococcus aureus (MRSA) and reported MIC values as low as $200 \mu \mathrm{g} / \mathrm{mL}$ [15]. Several studies have also reported good inhibitory activity for Australian plants against the gastrointestinal protozoal parasite Giardia duodenalis [16-18]. Furthermore, a recent study from our group reported notable antimicrobial activity for one of the species examined herein (B. obovata) $[19,20]$. However, all those studies examined extracts prepared under laboratory conditions using organic solvents and did not consider the traditional preparation methods.

Six plant species were selected for screening in our study, based on their ethnobotanical used. Buchanania obovata Engl. (family Anacardiaceae; commonly known as green plum and wild mango) is a small- to medium-sized tree native to the northern regions of the Northern Territory, Australia [19]. It produces small $(1-1.5 \mathrm{~cm}$ diameter) fleshy green fruit that are high in antioxidants. As well as being considered as nutritious food, the First Australians used B. obovata as a bacteriocide to treat skin diseases and as a wound antiseptic, as well as for treating eye infections and diarrhoea [9, 19]. Casuarina equisetifolia L. (family Casuarinaceae; commonly known as whistling tree and Australian pine tree) is native to northern regions of Australia, as well as Papua New Guinea, Southeast Asia, and the Pacific Islands. Its leaves and inner cambium are used as traditional antiseptics on Groote Eylandt [9]. Eucalyptus tetrodonta F. Muell. (family Myrtaceae; commonly known as Darwin stringybark and messmate) was also selected due to its traditional use as an antiseptic and to treat diarrhoea [9]. It is a medium-sized tree with rough fibrous bark. Several parts of the tree, including the inner bark, leaves, and kinos have been used traditionally as antibacterial medicines [9]. Planchonia careya (F. Muell.) R. Knuth. (family Lecythidaceae; commonly known as cocky apple and cockatoo apple) produces edible fruit that were prized by the First Australians. The cambium layer inside the bark and the leaves were used as medicines to treat skin diseases and 
diarrhoea [9]. The outer bark and the roots were also used as fish poison, indicating that they may also be useful in blocking cancer cell proliferation. Terminalia carpentariae C. T. White (family Combretaceae; commonly known as wild peach) is a small tree native to coastal regions of northern Australia. The edible fruit of this species have high antioxidant contents. The fruit, inner bark, and leaves were traditionally used to treat skin diseases and diarrhoea [9]. Similarly, Vigna vexillata (L.) A. Rich. (family Fabaceae; commonly known as wild cowpea) is a climbing plant with a wide distribution. It is native to tropical regions globally, including northern regions of Australia. The root is used as food, whilst the cambium layer and the leaves are used to treat skin disorders and diarrhoea by Groote Eylandt Aborigines [9]. The ethnobotanical uses of these plants indicate that they are good candidates for screening studies. Despite these, there are few reports confirming the bioactivities of these plants, and those studies that have been published invariably report on the activity of solvent extracts or essential oils. Studies testing these plants the way that they were originally used do not yet exist.

Diarrhoea may also be caused by nonbacterial pathogens. Gastrointestinal parasites, particularly protozoa (e.g., Cryptosporidium spp., Giardia duodenalis, Entamoeba histolytica, Blastocystis spp., and Cyclospora cayetanensis), also frequently cause diarrhoea. Entamoeba histolytica and G. duodenalis infections are relatively frequent, especially in socioeconomically depressed regions and in rural areas [20]. More than 280 million people per year are diagnosed with giardiasis internationally (WHO, http://www.who.int/ith/ diseases/giardiasis/en/), accounting for a significant health burden. Despite this, the effects of traditional medicines on protozoal pathogens have been relatively neglected in diarrhoeal studies, with only a few studies screening Australian medicinal plants against $G$. duodenalis $[12,16]$. Similarly, the effects of gastrointestinal viruses are poorly studied with very few studies reporting the potential of Australian plants to inhibit the replication of enteric viruses. The current study was undertaken to highlight plant species used by First Australian communities on Groote Eylandt against a panel of bacterial, protozoal, and viral gastrointestinal pathogens that cause diarrhoea. Furthermore, the antiproliferative activity of the extracts was also evaluated against a colorectal human cancer cell line as well as HeLa human cervical carcinoma cell line for comparison.

\section{Materials and Methods}

2.1. Study Site. Groote Eylandt is the largest island in the Gulf of Carpentaria and lies approximately $50 \mathrm{~km}$ off the coast of mainland Australia (Figure 1). It is within the Arnhem Land region of Australia and experiences tropical weather conditions for the entire year. Despite its proximity to the mainland and the close relationship of the Warnindhilyagwa people with the Nunggubuyu people on the mainland, the Warnindhilyagwa tribe have retained their own unique identity and much of their traditional knowledge.
2.2. Plant Species Selection. Few published resources on the traditional therapeutic use of plants by the First Australians exist, and we were only able to find a single ethnobotanical book devoted to the ethnobotany of Groote Eylandt [9]. That book was used as a resource to narrow the focus and create a list of twenty plants used traditionally by the Warnindhilyagwa people to treat gastrointestinal illnesses, with an emphasis on diarrhoeal diseases. Where the usage of a plant was ambiguous, the species was excluded. This list was used to compare information obtained from informal ethnobotanical surveys conducted with a panel of Warnindhilyagwa elders. Six female tribal elders reputed to be knowledgeable about traditional medicines were surveyed to further shortlist the plants for study. The ethnobotany surveys adhered to all ethical principles in the Code of Ethics of the International Society of Ethnobotany [21]. The survey participants were asked to name plants that they knew to be useful to treat diarrhoea. Only those plant species that were named by at least four of the six survey participants were selected for further study. This listing was compared to the shortlist obtained with reference to Levitt [9], and only species on both lists were included in our study. By this process, we narrowed the focus of the study to six species.

\subsection{Plant Material and Extraction. Gayangwa Lalara and} Gwen Lalara, elders of the Warnindhilyagwa tribe on Groote Eylandt, were consulted to identify local plant species that were traditionally used to treat gastrointestinal diseases by the Warnindhilyagwa people. Buchanania obovata Engl., Casuarina equisetifolia L., Eucalyptus tetrodonta F. Muell., Planchonia careya (F. Muell.) R. Knuth, Terminalia carpentariae C. T. White, and Vigna vexillata (L.) A. Rich. were highlighted as useful species to screen in this study. The tribal elders identified the trees, harvested (Figure 2(a)) and processed the plant material in November 2013 (Figure 2(b)), and prepared the decoctions (Figures 2(c) and 2(d)) used in these studies. The plant species highlighted and collected on Groote Eylandt were initially identified by the tribe elders involved in the collection. High-definition photographs were taken of the entire plant as well as various morphological features (leaves, flowers, etc.) for confirmation identity. Additionally, further samples were collected for identification purposes. The identity of each species was later confirmed by botanists at the Darwin Botanical Gardens from the collected material and the photographs. Voucher specimens of all collected species are stored in the School of Environment and Science, Griffith University. All decoctions were prepared by traditional methods immediately after harvesting. The traditional preparation method was the same for all the collected materials or each plant material. Trunks of immature plant specimens were cut, and the outer bark was scraped away with a knife. The inner cambium layers were carefully collected and processed separately. The cambium of each species was pounded with the inner wood from which it was removed to soften it for extraction, and the cambium was teased apart into strands by hand to increase the surface area for extraction. Each of the plant materials was placed into individual bowls, and 


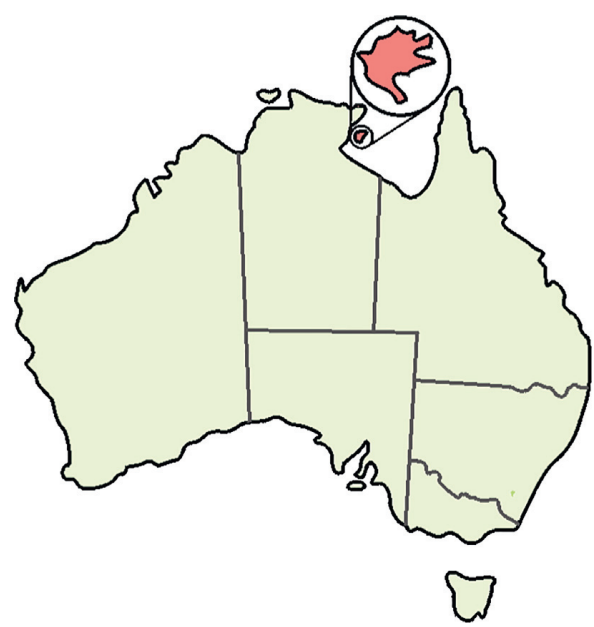

(a)

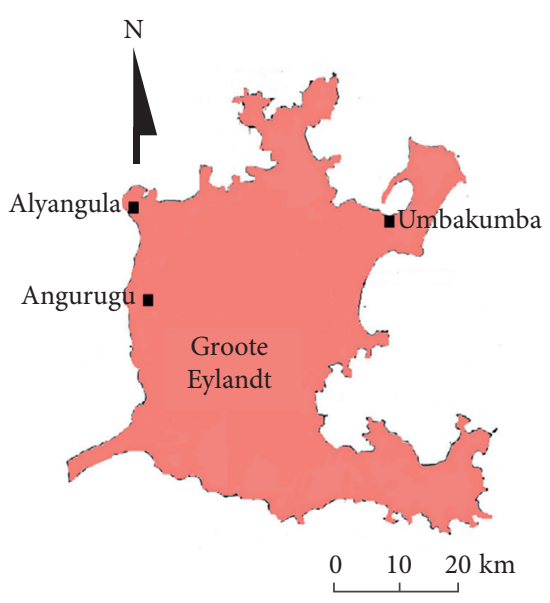

(b)

Figure 1: (a) The location of Groote Eylandt in relation to mainland Australia and (b) a map of Groote Eylandt showing the main settlements.

enough deionised water was added to just cover the plant material. The plant material was allowed to extract for an hour, and the decoction was decanted from the cambium into clean plastic bottles. The bottles were stored on ice for transport to the laboratory where they were filtered through Whatman No. 54 filter paper under vacuum. Aliquots $(1 \mathrm{~mL})$ of each decoction were dried in preweighed $1.5 \mathrm{~mL}$ microfuge tubes to determine the mass of extracted plant material $/ \mathrm{mL}$ of decoction. The remaining aliquots of each extract were stored at $-30^{\circ} \mathrm{C}$ until use.

2.4. Qualitative Phytochemical Studies. Qualitative phytochemical analysis of the plant extracts for the presence of alkaloids, anthraquinones, cardiac glycosides, flavonoids, phenolic compounds, phytosterols, saponins, tannins, and triterpenoids was evaluated by standard assays [22-24].

2.5. Evaluation of Antimicrobial Activity. The bioassays used to screen and quantify therapeutic properties examined in this study followed the methods of Rabadeaux et al. [25].

2.5.1. Test Bacteria. Aeromonas hydrophila (ATCC 7966), Bacillus cereus (ATCC 14579), Escherichia coli (ATCC O157 H7), Shigella sonnei (ATCC 25931), Staphylococcus aureus (ATCC 157293), and Streptococcus pyogenes (ATCC 12384) were obtained from the American Type Culture Collection (ATCC), USA. The clinical bacterial strains Alcaligenes faecalis, Bacillus subtilis, Citrobacter freundii, Clostridium perfringens, Salmonella salford, Salmonella newport, Staphylococcus epidermidis, and Yersinia enterocolitica were obtained from the School of Environment and Science teaching laboratory at Griffith University. Apart from C. perfringens, all strains were subcultured and maintained aerobically in nutrient broth and on nutrient agar at $37^{\circ} \mathrm{C}$ (Oxoid Ltd., Australia). Clostridium perfringens was grown and maintained in thioglycolated liquid media (Oxoid Ltd., Australia) under induced anaerobic conditions in anaerobic jars using AnaeroGen $^{\mathrm{TM}}$ 3.5 L atmospheric generation systems (Thermo Scientific).

2.5.2. Standard Antibiotics. Penicillin G (potency of $1440-1680 \mu \mathrm{g} / \mathrm{mg})$, chloramphenicol ( $\geq 98 \%$ purity by HPLC), erythromycin (potency $\geq 850 \mu \mathrm{g} / \mathrm{mg}$ ), ciprofloxacin ( $\geq 98 \%$ purity by HPLC), and tetracycline ( $\geq 95 \%$ purity by HPLC) were purchased from Sigma-Aldrich (Australia) and were used as controls for the microplate liquid dilution assays. Standard discs of ampicillin $(10 \mu \mathrm{g})$ and chloramphenicol $(10 \mu \mathrm{g})$ were obtained from Oxoid Ltd., Australia, and served as positive controls for the disc diffusion assay.

2.5.3. Evaluation of Antimicrobial Activity. The antimicrobial activity of all the plant extracts was initially determined using a modified disc diffusion method [25]. Briefly, bacterial cultures were grown in nutrient broth until they reached a count of approximately $10^{8}$ cells $/ \mathrm{mL}$; then, $100 \mu \mathrm{L}$ of each suspension was spread uniformly onto individual agar plates. The extracts were screened for antibacterial activity by applying $6 \mathrm{~mm}$ sterilised filter paper discs infused with $10 \mu \mathrm{L}$ of the test sample onto the agar surface and incubating for 24 hours at $37^{\circ} \mathrm{C}$. Following incubation, the diameters of the inhibition zones were measured to the closest whole millimetre. Each antimicrobial assay was performed three times in triplicate $(n=9)$, and mean values were determined. Standard discs of ampicillin $(10 \mu \mathrm{g})$ and chloramphenicol $(10 \mu \mathrm{g})$ served as positive controls. Discs infused with $10 \mu \mathrm{L}$ of distilled water were included as negative controls.

2.5.4. Minimum Inhibitory Concentration (MIC) Determination. The minimum inhibitory concentration for each extract was determined using two methods. Colorimetric liquid dilution MIC assays were used as they are 


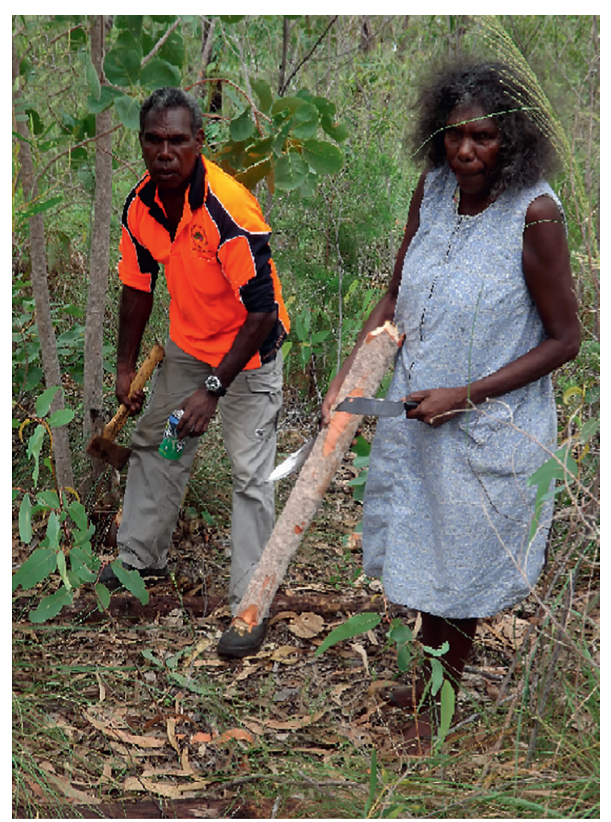

(a)

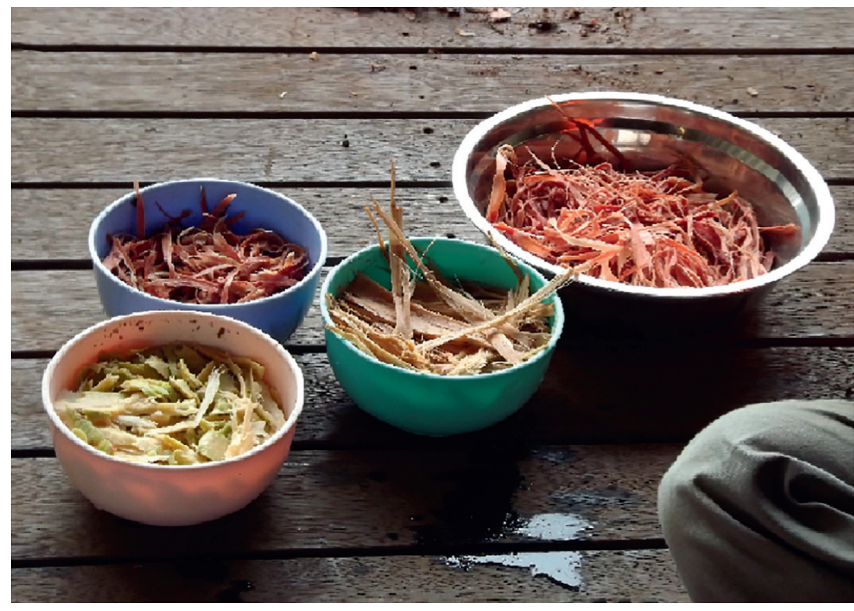

(c)

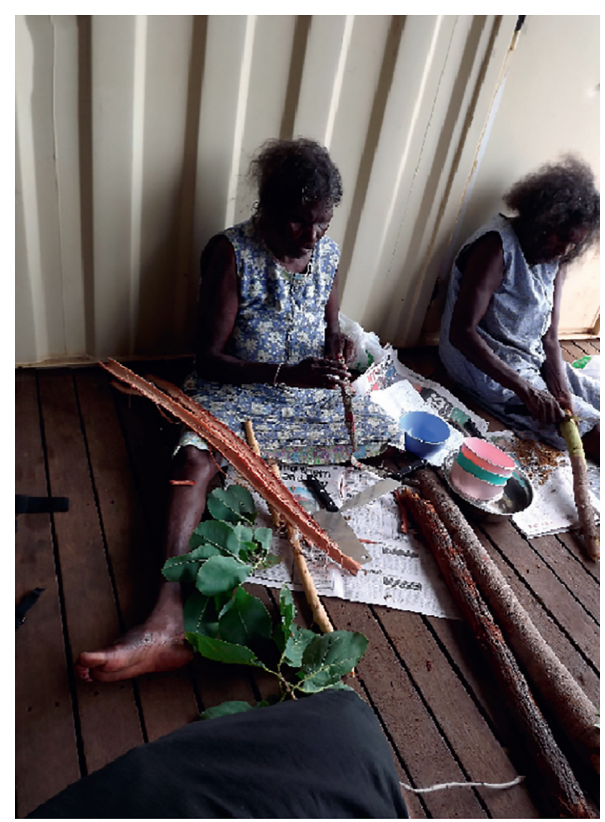

(b)

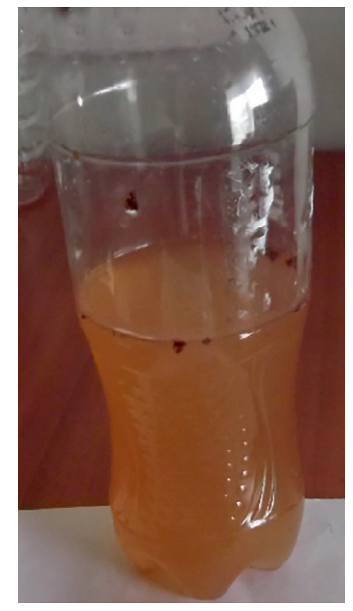

(d)

FIGURE 2: (a) Collection of plant materials on Groote Eylandt by members of the Warnindhilyagwa tribe, (b) processing of the plant material by Warnindhilyagwa elders Gayangwa Lalara and Gwen Lalara, (c) preparation of the medicines by traditional methods, and (d) an example of the traditionally prepared decoctions.

sensitive measures of bacterial growth inhibitory activity [12]. This is a commonly used method of quantifying bacterial growth inhibition efficacy, allowing for comparisons with other studies. A solid-phase agar disc diffusion assay was also used in this study as a comparison and as a closer representation of solid-phase infections.

2.5.5. Broth Microdilution MIC Assay. The MICs of the extracts were evaluated by standard broth microdilution methods [26, 27]. Briefly, overnight bacterial cultures were added dropwise to fresh nutrient broth, and the turbidity was visually adjusted to produce a McFarland number 1 standard culture. This was subsequently diluted 1 in 50 with nutrient broth, resulting in the MIC assay inoculum culture. A volume of $100 \mu \mathrm{L}$ sterile broth was added to all wells of a 96-well plate. Test extracts or control antibiotics $(100 \mu \mathrm{L})$ were then added to the top row of each plate, and doubling dilutions were prepared in each column of wells by transferring $100 \mu \mathrm{L}$ from the top well to the next well in each column, etc. A growth control (without the extract) and a sterile control (without the inoculum) were included on each plate. A volume of $100 \mu \mathrm{L}$ of respective bacterial culture was added to all wells except the sterile control wells and incubated at $37^{\circ} \mathrm{C}$ for $24 \mathrm{~h}$. $p$-Iodonitrotetrazolium violet (INT) was obtained from Sigma-Aldrich, Australia, and dissolved in sterile deionised water to prepare a $0.2 \mathrm{mg} / \mathrm{mL}$ INT solution. A $40 \mu \mathrm{L}$ volume of this solution was added into all wells, and the plates were incubated for further $6 \mathrm{~h}$ at $37^{\circ} \mathrm{C}$. 
Following incubation, the MIC was visually determined as the lowest dose at which colour development was inhibited.

2.5.6. Disc Diffusion MIC Quantification. The minimum inhibitory concentration (MIC) of the extracts was also determined by disc diffusion assays following the methods of Rabadeaux et al. [25] across a range of doses, and graphs of the zone of inhibition versus the natural log of the concentration were used to calculate the MIC values of each extract.

\subsection{Inhibitory Bioactivity against Giardia duodenalis Trophozoites}

2.6.1. Parasite Culture. The Giardia duodenalis S-2 (sheep strain 2) trophozoite strain used in this study was a gift from Professor Ann McDonnell, Griffith University, Australia. The trophozoites were maintained and subcultured anaerobically at $37^{\circ} \mathrm{C}$ in TYI-S-33 growth media supplemented with $1 \%$ bovine bile (Sigma), 10\% Serum Supreme (Cambrex Bioproducts), and $200 \mathrm{IU} / \mathrm{mL}$ penicillin $/ 200 \mu \mathrm{g} / \mathrm{mL}$ streptomycin (Invitrogen, USA). Confluent mid-log phase cultures were passaged every 2 days by chilling the cultures on ice for a minimum of $10 \mathrm{~min}$ followed by vortexing to dislodge the adherent trophozoites from the walls of the culture vessel. Fresh culture media $(5 \mathrm{~mL})$ were seeded with approximately $1 \times 10^{5}$ trophozoites for each passage.

2.6.2. Evaluation of Anti-Giardial Activity. Anti-Giardial activity was determined as previously described [25]. Briefly, $30 \mu \mathrm{L}$ of the test extracts or the vehicle solvent or culture media (for the negative controls) was added to trophozoite suspensions $\left(70 \mu \mathrm{L}\right.$ containing approximately $1 \times 10^{5}$ trophozoites) in 96-well plates. The plates were incubated anaerobically at $37^{\circ} \mathrm{C}$ for $12 \mathrm{~h}$ in a humidified anaerobic atmosphere. CellTiter $96^{\circledR}$ AQueous One Solution Cell Proliferation Assay Reagent $(20 \mu \mathrm{L}$; Promega) was added to each well, and the plates were incubated for further $3 \mathrm{~h}$. The absorbances were recorded at $490 \mathrm{~nm}$ using a Molecular Devices, SpectraMax M3 plate reader. All tests were performed three times in triplicate $(n=9)$. The antiproliferative activity of each test was expressed as a percentage of the negative untreated control.

\subsection{Screen for Antiviral Bioactivity}

2.7.1. Viral and Bacterial Stocks. The MS2 bacteriophage and $\mathrm{F}+\mathrm{Amp}+E$. coli used in this study were purchased from the American Type Culture Collection (ATCC). The clinical Staphylococcus aureus strain used in this study was obtained from Michelle Mendell, Griffith University. A volume of $30 \mathrm{~mL}$ of nutrient broth (Oxoid, Australia) containing $100 \mu \mathrm{g} /$ $\mathrm{mL}$ ampicillin (Sigma-Aldrich, Australia) was inoculated with $1 \mathrm{~mL}$ of $\mathrm{F}+\mathrm{Amp}+$ E. coli culture and incubated for $2 \mathrm{~h}$ at $37^{\circ} \mathrm{C}$ to reach the log phase. A volume of $1 \mathrm{~mL}$ MS2 virus stock solution (containing approximately $10^{8}$ plaque-forming units) was subsequently added to the bacterial culture and incubated overnight at $37^{\circ} \mathrm{C}$. The following day, the bacterial cells were centrifuged at $4000 \mathrm{rpm}$ for $10 \mathrm{~min}$, and the supernatant (containing free MS2 phage) was collected, passed through a $22 \mu \mathrm{m}$ Sarstedt filter, and stored at $4^{\circ} \mathrm{C}$ until use.

2.7.2. Soft-Agar Overlay. Immediately prior to the MS2 phage assay, soft-agar overlay containing $0.7 \%(\mathrm{w} / \mathrm{v}) \mathrm{nu}-$ trient agar, $1 \%(\mathrm{w} / \mathrm{v})$ glucose, $1 \%(\mathrm{w} / \mathrm{v}) \mathrm{CaCl}_{2}$, and $1 \%(\mathrm{w} / \mathrm{v})$ $\mathrm{MgSO}_{4}$ was prepared and used immediately for the MS2 plaque inhibition assay described in the following.

2.7.3. MS2 Plaque Inhibition Assay. The MS2 bacteriophage plaque reduction assay was performed as previously described [28]. Briefly, $490 \mu \mathrm{L}$ of each plant extract dilution was inoculated with $10 \mu \mathrm{L}$ of MS2 virus (containing approximately $10^{10}$ plaque-forming units $/ \mathrm{mL}$ ) and incubated overnight at $4^{\circ} \mathrm{C}$. The solution was added to $500 \mu \mathrm{L}$ Staphylococcus aureus and incubated at $37^{\circ} \mathrm{C}$ for $20 \mathrm{~min}$. The mixture was subsequently added to $3 \mathrm{~mL}$ soft-agar overlay and poured over premade agar plates $(2.8 \% \mathrm{w} / \mathrm{v}$ nutrient agar). Once set, the plates were incubated overnight at $37^{\circ} \mathrm{C}$. The following day, the number of plaques was counted and expressed as the \% untreated control. Extract dilutions were tested, and $\mathrm{IC}_{50}$ was determined by linear regression. $\mathrm{Nu}-$ trient broth was used as a negative control, whilst a C. sinensis water extract and UV irradiation (microwave of $10 \mu \mathrm{L}$ for $4 \times 30 \mathrm{sec}$ ) were used as positive controls.

\subsection{Screen for Anticancer Bioactivity}

2.8.1. Cancer Cell Lines. In previous studies in our group, we have studied the antiproliferative activity of plant extracts against $\mathrm{Caco} 2$ and HeLa (American Type Culture Collection, USA) carcinoma cell lines [25]. We followed the methods described in that study to maintain and passage the cells at $37^{\circ} \mathrm{C}, 5 \% \mathrm{CO}_{2}$ in a humidified atmosphere, until approximately $80 \%$ confluent.

2.8.2. Evaluation of Cancer Cell Antiproliferative Activity. We followed the methods of Rabadeaux et al. [25] to evaluate the antiproliferative activity of the plant extracts. Briefly, $70 \mu \mathrm{L}$ aliquots of the individual carcinoma cell suspensions were aspirated into the wells of a 96 -well plate. $30 \mu \mathrm{L}$ of the plant extract dilutions or controls was added to individual wells, and the plates were incubated at $37^{\circ} \mathrm{C}, 5 \% \mathrm{CO}_{2}$. Cisplatin $(50 \mu \mathrm{g} / \mathrm{mL}$; Sigma-Aldrich, Australia) was used as a positive control, and fresh media were included as a negative control. Following 12-hour incubation, $20 \mu \mathrm{L}$ of CellTiter 96 AQueous One Solution (Promega, Australia) was added to each well. The absorbance was recorded at $490 \mathrm{~nm}$ as a measure of cellular proliferation following further $3 \mathrm{~h}$ incubation at $37^{\circ} \mathrm{C}$. The antiproliferative activity of each test was recorded as a percentage of the negative control.

2.8.3. Toxicity Evaluation. Two assay methods were used to assess the toxicity of the individual samples. The Artemia 
lethality assay (ALA) was utilised for rapid preliminary toxicity screening, whilst an MTS-based cellular viability assay was used as a cellular evaluation of toxicity.

2.8.4. Artemia franciscana Nauplii Toxicity Screening. Toxicity was tested using a modified $A$. franciscana nauplii lethality assay $[29,30]$. Briefly, $400 \mu \mathrm{L}$ of seawater containing approximately 52 (mean $51.8 \quad n=125, \quad$ SD 11.2 ) A. franciscana nauplii was added to wells of a 48 -well plate and immediately used for bioassay. A volume of $400 \mu \mathrm{L}$ of diluted plant extracts or the reference toxin $(1000 \mu \mathrm{g} / \mathrm{mL}$ potassium dichromate) was transferred to the wells and incubated at $25 \pm 1^{\circ} \mathrm{C}$ under artificial light (1000 lux). A negative control ( $400 \mu \mathrm{L}$ seawater) was included on each plate. All treatments and controls were performed three times $(n=9)$. The wells were checked at regular intervals, and the number of dead was counted. After $24 \mathrm{~h}$, all nauplii were sacrificed and counted to determine the total \% mortality per well. LC 50 with $95 \%$ confidence limits for each treatment was calculated using probit analysis.

2.8.5. Cell Viability Assays. The decoctions were also screened for toxicity against human primary dermal fibroblasts (HDF). The HDF cells were obtained from the American Type Culture Collection (ATCC PCS-201-012) and were cultured and maintained in Dulbecco's modified eagle medium (DMEM; Thermo Fisher Scientific, Australia), supplemented with $10 \%$ fetal calf serum (Invitrogen), $50 \mu \mathrm{g} / \mathrm{mL}$ streptomycin (Sigma-Aldrich,
Australia), and $50 \mathrm{IU} / \mathrm{mL}$ penicillin (Sigma-Aldrich, Australia) by standard methods [31,32]. Briefly, the cells were maintained as monolayers in $7 \mathrm{~mL}$ flasks at $37^{\circ} \mathrm{C}, 5 \%$ $\mathrm{CO}_{2}$ in a humidified atmosphere, until approximately $80 \%$ confluent. Once confluency was achieved, $1 \mathrm{~mL}$ of trypsin (Sigma-Aldrich, Australia) was added to the culture flasks and incubated at $37^{\circ} \mathrm{C}, 5 \% \mathrm{CO}_{2}$, for $15 \mathrm{~min}$ to dislodge the HDF cells. The cell suspensions were then transferred to a $10 \mathrm{~mL}$ centrifuge tube and sedimented by centrifugation. The supernatant was discarded, and the cells were resuspended in $9 \mathrm{~mL}$ of fresh media. Aliquots of the resuspended cells $(70 \mu \mathrm{L}$ containing approximately 5000 cells) were added to individual wells of a 96-well plate. A volume of $30 \mu \mathrm{L}$ of the test extracts or cell media (for the negative control) was added to individual wells, and the plates were incubated at $37^{\circ} \mathrm{C}, 5 \% \mathrm{CO}_{2}$ for $24 \mathrm{~h}$ in a humidified atmosphere. All extracts were screened at $200 \mu \mathrm{g} /$ $\mathrm{mL}$. The cells were then washed in PBS ( $\mathrm{pH}$ 7.2) to remove interference due to sample colour. A volume of $20 \mu \mathrm{L}$ of CellTiter 96 AQueous One Solution (Promega) was subsequently added to each well, and the plates were incubated for further $3 \mathrm{~h}$. Absorbances were recorded at a test wavelength of $540 \mathrm{~nm}$ and a blank wavelength of $690 \mathrm{~nm}$ using a Molecular Devices, SpectraMax M3 plate reader. All tests were performed in at least triplicate, and triplicate controls were included on each plate. The \% cellular viability of each test was calculated using the following formula:

$$
\% \text { cellular viability }=\frac{\text { Abs test sample }-(\text { mean Abs control }- \text { mean Abs blank })}{(\text { mean Abs control }- \text { mean Abs blank })} .
$$

Cellular viability $\leq 50 \%$ of the untreated control indicated toxicity, whereas extracts or controls with $>50 \%$ untreated control viability were deemed to be nontoxic.

2.8.6. Statistical Analysis. Data are expressed as the mean\pm SEM of three independent experiments, each with three internal replicates $(n=9)$. One-way ANOVA followed by Tukey's post hoc analysis were used to calculate statistical significance between control and treated groups with a $P$ value $<0.01$ considered to be statistically significant.

\section{Results}

3.1. Qualitative Phytochemical Screening of the Traditional Medicines. The decoctions prepared traditionally from the individual cambiums yielded preparations with varying concentrations (Table 1). The concentrations of the decoctions were relatively consistent (generally $1-4 \mathrm{mg} / \mathrm{mL}$ ) for most of the plant species. However, substantially higher concentrations were noted for the $B$. obovata and T. carpentariae decoctions $(7.8$ and $10.2 \mathrm{mg} / \mathrm{mL}$, respectively). Phytochemical studies (Table 1) showed that all the decoctions contained similar classes of phytochemicals. All contained high relative abundances of phenolics and (with the exception of $V$. vexillata) moderate to high levels of tannins. Flavonoids were present in low to moderate abundance across all extracts, and saponins were noted in most decoctions (except $B$. obovata and $V$. vexillata). All other phytochemical classes were either missing or in low abundance in all decoctions.

3.2. Antibacterial Activity. Aliquots $(10 \mu \mathrm{L})$ of each extract were tested in the disc diffusion assay against panels of Gram-negative (Figure 3) and Gram-positive bacteria (Figure 4). All the decoctions possessed broad-spectrum inhibitory activity. Indeed, the $B$. obovata, E. tetrodonta, $P$. careya, and $V$. vexillata decoctions each inhibited all the Gram-negative bacteria species tested. The E. coli strain was resistant to the C. equisetifolia and T. carpentariae decoctions, although all other Gram-negative bacterial species tested were inhibited by these decoctions. It is noteworthy that the E. coli strain tested in this study was also relatively resistant to the other decoctions as only relatively small ZOIs were measured against those decoctions. Therefore, it is 
TABLE 1: The concentration $(\mathrm{mg} / \mathrm{mL})$ and qualitative phytochemical screening evaluations of the plant decoctions.

\begin{tabular}{|c|c|c|c|c|c|c|}
\hline $\begin{array}{l}\text { Decoction concentration } \\
(\mathrm{mg} / \mathrm{mL})\end{array}$ & $\begin{array}{l}\mathrm{BO} \\
7.8\end{array}$ & $\begin{array}{l}\mathrm{CE} \\
3.4\end{array}$ & $\begin{array}{l}\text { ET } \\
2.9\end{array}$ & $\begin{array}{l}\mathrm{PC} \\
4.2\end{array}$ & $\begin{array}{c}\mathrm{TC} \\
10.2\end{array}$ & $\begin{array}{l}\text { VV } \\
1.2\end{array}$ \\
\hline Total phenolics & +++ & ++ & +++ & +++ & +++ & ++ \\
\hline Water-soluble phenolics & +++ & +++ & ++ & +++ & +++ & + \\
\hline Water-insoluble phenolics & +++ & +++ & + & +++ & ++ & ++ \\
\hline Cardiac glycosides & - & - & - & - & - & - \\
\hline Saponins & - & ++ & ++ & + & ++ & - \\
\hline Triterpenoids & - & - & - & - & ++ & - \\
\hline Phytosterols & - & - & - & - & - & - \\
\hline Alkaloids (Mayer test) & - & - & - & - & + & - \\
\hline Alkaloids (Wagner test) & - & - & - & - & - & - \\
\hline Flavonoids & ++ & + & + & + & ++ & + \\
\hline Tannins & +++ & ++ & ++ & +++ & +++ & - \\
\hline Anthraquinones & - & - & - & - & - & - \\
\hline
\end{tabular}

+++ indicates a large response; ++ indicates a moderate response; + indicates a minor response; - indicates no response in the assay; $\mathrm{BO}=$ B. obovata extract; $\mathrm{CE}=C$. equisetifolia extract; $\mathrm{ET}=E$. tetrodonta extract; $\mathrm{PC}=P$. careya extract; $\mathrm{TC}=T$. carpentariae extract; $\mathrm{VV}=V$. vexillata extract.

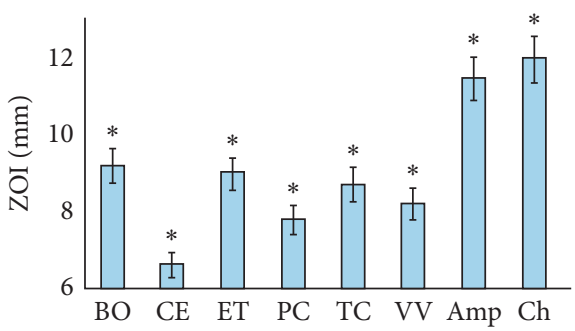

(a)

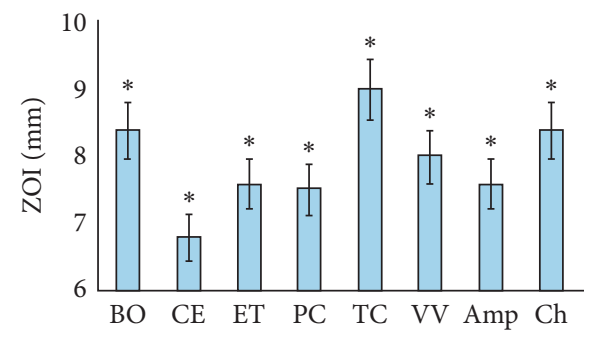

(c)

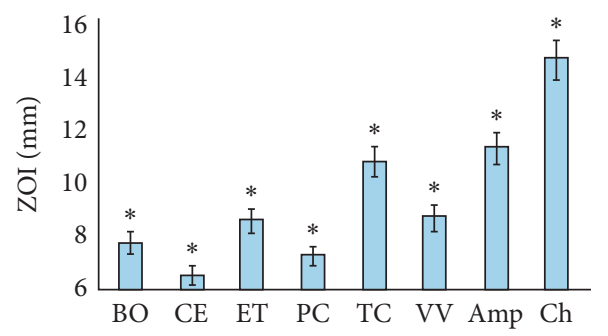

(e)

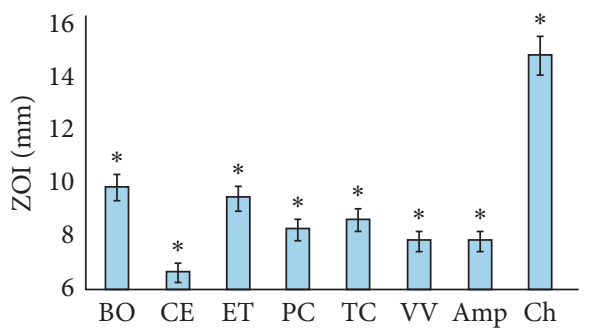

(b)

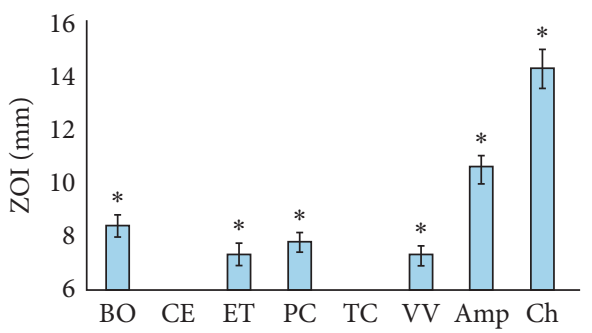

(d)

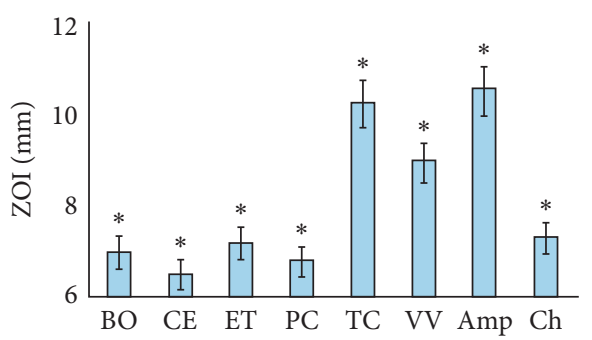

(f)

Figure 3: Continued. 


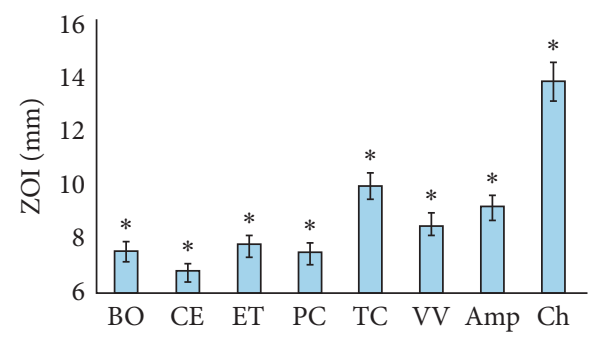

(g)

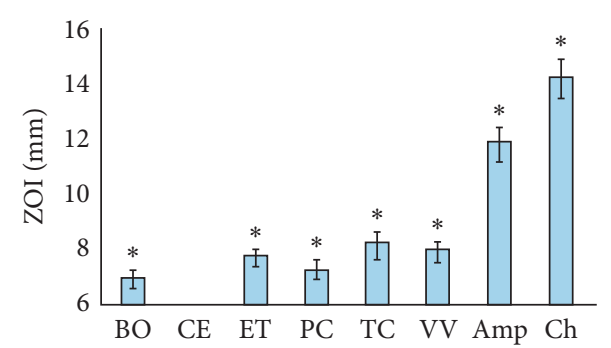

(h)

FIgURE 3: Growth inhibitory activity of the decoctions and ampicillin and chloramphenicol controls (10 $\mu \mathrm{g})$ against the Gram-negative bacteria (a) A. faecalis, (b) A. hydrophila, (c) C. freundii, (d) E. coli, (e) S. salford, (f) S. newport, (g) S. sonnei, and (h) Y. enterocolitica measured as ZOIs $(\mathrm{mm}) . \mathrm{BO}=B$. obovata extract; $\mathrm{CE}=C$. equisetifolia extract; $\mathrm{ET}=E$. tetrodonta extract; $\mathrm{PC}=P$. careya extract; $\mathrm{TC}=T$. carpentariae extract; $\mathrm{VV}=V$. vexillata extract; $\mathrm{Amp}=$ ampicillin; $\mathrm{Ch}=$ chloramphenicol. Results are expressed as mean $\pm \mathrm{SEM}$ at three determinations in triplicate $(n=9) .{ }^{*}$ indicates results that are significantly different to the untreated control $(P<0.01)$.

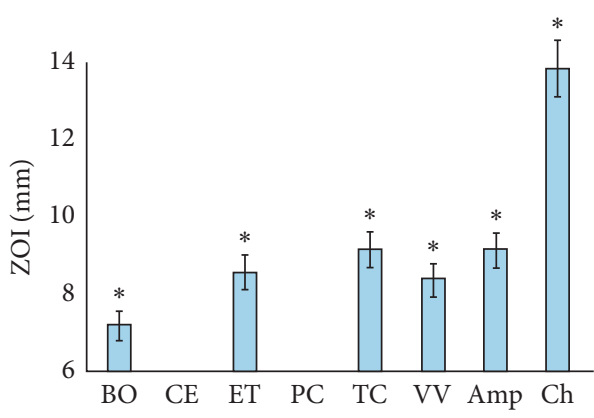

(a)

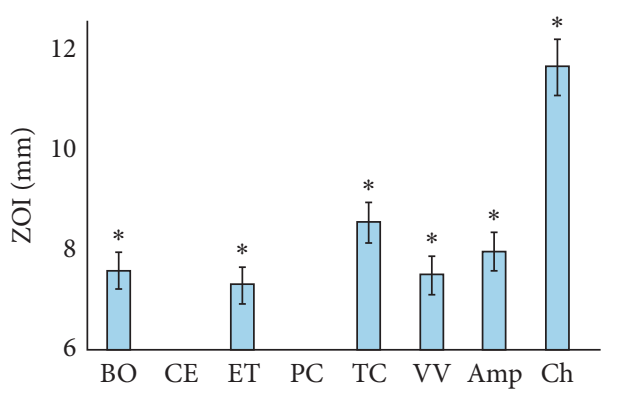

(c)

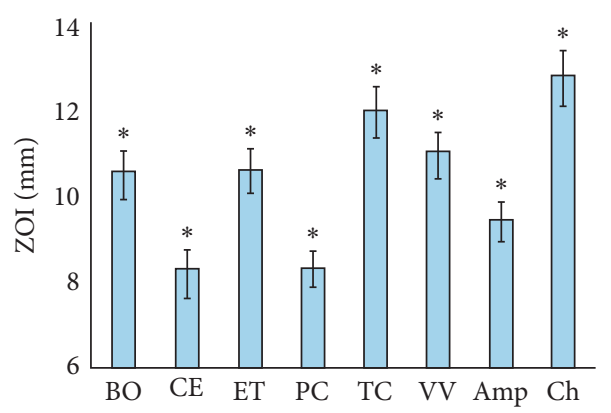

(e)

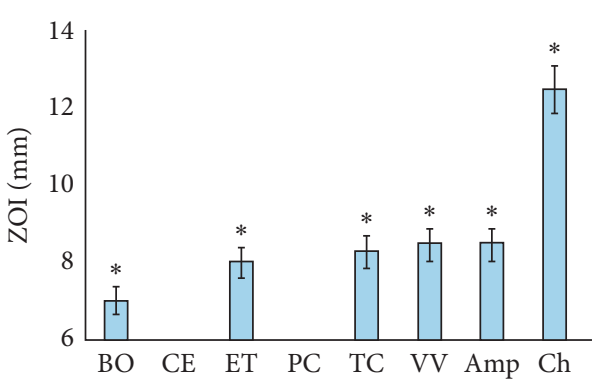

(b)

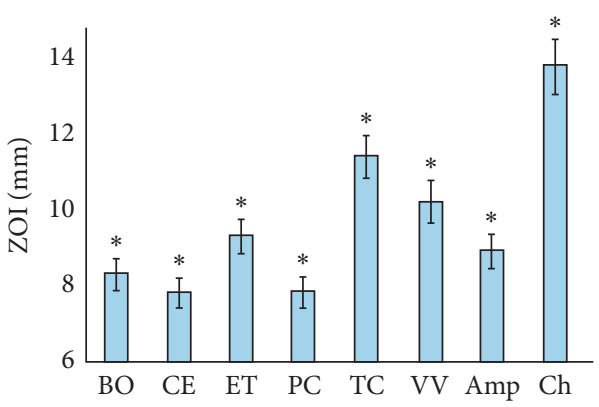

(d)

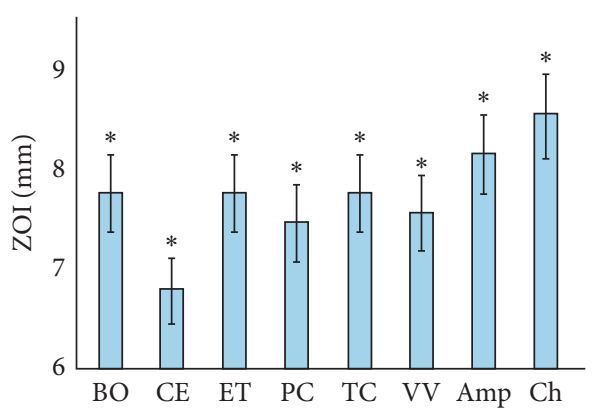

(f)

Figure 4: Growth inhibitory activity of the decoctions and ampicillin and chloramphenicol controls (10 $\mu \mathrm{g})$ against the Gram-positive bacteria (a) B. cereus, (b) B. subtilis, (c) C. perfringens, (d) S. aureus, (e) S. epidermidis, and (f) S. pyogenes measured as ZOIs (mm). $\mathrm{BO}=B$. obovata extract; $\mathrm{CE}=C$. equisetifolia extract; $\mathrm{ET}=$ E. tetrodonta extract; $\mathrm{PC}=P$. careya extract; $\mathrm{TC}=T$. carpentariae extract; $\mathrm{VV}=V$. vexillata extract; $\mathrm{Amp}=$ ampicillin; $\mathrm{Ch}=$ chloramphenicol. Results are expressed as mean $\pm \mathrm{SEM}$ at three determinations in triplicate $(n=9) .{ }^{*}$ indicates results that are significantly different to the untreated control $(P<0.01)$. 
possible that this is a particularly resistant strain, and other E. coli strains may be inhibited by these decoctions. Further work is required to test these decoctions against other E. coli strains.

The B. obovata, E. tetrodonta, T. carpentariae, and $V$. vexillata decoctions were also broad-spectrum inhibitors of Gram-positive bacteria, each inhibiting all of the bacteria tested. In contrast, $C$. equisetifolia and $P$. careya decoctions inhibited the growth of $50 \%$ of the Gram-positive bacterial species tested. Interestingly, both Bacillus spp. and C. perfringens were resistant to these decoctions. As both Bacillus and Clostridium genera can form endospores, it is possible that these decoctions may still be effective against the planktonic form of the bacterium yet be ineffective against the endospores. Thus, even if they kill the vegetative cells, the bacterium may regrow from spores, thus masking any inhibition. Future studies are required to specifically test these decoctions against both vegetative cells and endospores.

The relative antimicrobial potency was further evaluated by determining the MIC values (Table 2) for each extract against the bacterial species which were susceptible in the decoctions. With some notable exceptions, most of these decoctions were effective at inhibiting microbial growth at low concentrations, with liquid dilution MIC values against the bacterial species that they inhibited generally substantially $<1000 \mu \mathrm{g} / \mathrm{mL}$, indicating the noteworthy antimicrobial activity of these preparations. The $T$. carpentariae decoction was a particularly good inhibitor of bacterial growth, with MIC values $<1000 \mu \mathrm{g} / \mathrm{mL}$ against all bacteria tested. $V$. vexillata was also a good antibacterial agent against most bacteria. Only E. coli had an MIC $>1000 \mu \mathrm{g} / \mathrm{mL}$ against this decoction, and its MIC of $1712 \mu \mathrm{g} / \mathrm{mL}$ still indicates moderate activity. In contrast to the other decoctions, the C. equisetifolia preparation had only low to moderate antibacterial activity, with MIC values $1750-2850 \mu \mathrm{g} / \mathrm{mL}$. Whilst these values indicate that the C. equisetifolia decoction may still be useful in treating gastrointestinal bacterial infections, they would be less effective than the other extracts.

3.3. Anti-Giardial Activity. The extracts were screened for their ability to inhibit Giardia duodenalis growth (Figure 5(a)). Whilst the C. equisetifolia, E. tetrodonta, $T$. carpentariae, and $V$. vexillata decoctions displayed significant inhibitory activity, the level of inhibition was relatively low (20-40\% inhibition of control proliferation), indicating only weak growth inhibitory activity. As the inhibition of $G$. duodenalis growth did not exceed $50 \%$ at any concentration tested for these preparations, it was not possible to determine $\mathrm{IC}_{50}$ values for these extracts (Table 2). The B. obovata decoction also displayed apparent inhibition (approximately 7\% inhibition) of $G$. duodenalis growth. However, this inhibition was not statistically significant.

3.4. Inhibition of MS2 Phage Production. Whilst MS2 phage is not infective in humans, it has previously been used as a safe and rapid test for antiviral activity against other human- infective RNA viruses $[28,33]$. Thus, the MS2 phage plaque reduction assay may indicate inhibitory activity against gastrointestinal viral pathogens such as Norovirus. It was therefore used in this study as a preliminary screen for potential gastrointestinal antiviral activity. Four of the decoctions tested in this study displayed strong antiviral activity in this bioassay. The T. carpentariae decoction was a particularly good inhibitor of MS2 reproduction, inhibiting MS2 phage production by approximately $98 \%$. This is noteworthy and is substantially more potent than the C. sinensis positive control ( $90 \%$ inhibition). The T. carpentariae decoction was also tested across a range of concentrations and an $\mathrm{IC}_{50}$ of $427 \mu \mathrm{g} / \mathrm{mL}$ was determined, indicating strong antiviral activity. The $B$. obovata, E. tetrodonta, and $V$. vexillata decoctions were also good inhibitors of MS2 phage replication, inhibiting viral replication by $60-80 \%$. The $\mathrm{IC}_{50}$ values determined for these decoctions (2283, 1140, and $2950 \mu \mathrm{g} / \mathrm{mL}$, respectively) (Table 2) also indicate noteworthy activity. In contrast, the $C$. equisetifolia and $P$. careya decoctions were completely devoid of anti-MS2 phage activity.

3.5. Inhibition of Cancer Cell Proliferation. All decoctions were tested against 2 cancer cell lines (Caco2 human colorectal carcinoma cells, Figure 6(a); HeLa human cervical cancer cells, Figure 6(b)) to determine their effects on cell proliferation. The $\mathrm{Caco} 2$ cell line was selected as it is a colorectal line and thus is consistent with intestinal disease. The HeLa cells were chosen as a comparison as they are well studied and allow for comparisons with other reports. Interestingly, with the exception of B. obovata, all of the decoctions significantly inhibited the proliferation of both Caco 2 and HeLa carcinoma cells compared to the proliferation of the untreated cells. T. carpentariae was a particularly strong inhibitor of $\mathrm{Caco} 2$ growth, inhibiting proliferation by approximately $80 \%$ compared to the untreated control cell growth (Figure 6(a)). All the other decoctions (except B. obovata) also significantly inhibited Caco 2 proliferation, albeit by substantially lower levels (15-30\%). However, it is noteworthy that the T. carpentariae decoction was substantially more concentrated than the other preparations, and this may account for its higher apparent activity. Inhibition of $\mathrm{Caco} 2$ growth by the T. carpentariae decoction was dose-dependent, and an $\mathrm{IC}_{50}$ value of $885 \mu \mathrm{g} / \mathrm{mL}$ was determined by screening the decoction across a range of doses. We were unable to determine the $\mathrm{IC}_{50}$ values of any of the other preparations against Caco 2 as the cell proliferation did not decrease below $50 \%$ at any concentration tested.

With the exception of the $B$. obovata preparation, the decoctions were also potent inhibitors of HeLa cell proliferation (Figure 6(b)) and were generally more potent inhibitors against this cell line than against Caco2. As for Caco 2 proliferation, the $T$. carpentariae decoction was the most potent inhibitor of HeLa cell proliferation (Figure 6(b), Table 2). Indeed, the T. carpentariae decoction blocked $100 \%$ of the HeLa proliferation during the screening experiment, and an $\mathrm{IC}_{50}$ of $85 \mu \mathrm{g} / \mathrm{mL}$ was calculated when the decoction 
TABLE 2: The MIC values $(\mu \mathrm{g} / \mathrm{mL})$ of the decoctions against bacteria, $\mathrm{IC}_{50}$ values $(\mu \mathrm{g} / \mathrm{mL})$ against $G$. duodenalis, MS2 phage, and Caco2 and HeLa cancer cell lines, and $\mathrm{LC}_{50}$ values $(\mu \mathrm{g} / \mathrm{mL})$ for $A$. franciscana nauplii and HDF bioassays.

\begin{tabular}{|c|c|c|c|c|c|c|c|c|c|}
\hline & \multirow{2}{*}{\multicolumn{2}{|c|}{ Bioassay }} & \multicolumn{6}{|c|}{ Plant species } & \multirow{2}{*}{ Positive control* } \\
\hline & & & $\mathrm{BO}$ & $\mathrm{CE}$ & ET & PC & TC & VV & \\
\hline \multirow{16}{*}{ Gram-negative bacteria } & \multirow{2}{*}{ A. faecalis } & DD MIC & 724 & 2850 & 854 & 1550 & 966 & 1280 & \multirow{2}{*}{0.31} \\
\hline & & LD MIC & 557 & 1938 & 504 & 977 & 628 & 870 & \\
\hline & \multirow{2}{*}{ A. hydrophila } & DD MIC & 645 & 3280 & 798 & 1274 & 858 & 1453 & \multirow{2}{*}{0.31} \\
\hline & & LD MIC & 387 & 2230 & 487 & 905 & 532 & 1090 & \\
\hline & \multirow{2}{*}{ C. freundii } & DD MIC & 972 & 3168 & 1357 & 1260 & 650 & 1086 & \multirow{2}{*}{0.63} \\
\hline & & LD MIC & 603 & 2839 & 923 & 1285 & 351 & 900 & \\
\hline & \multirow{2}{*}{ E. coli } & DD MIC & 1228 & - & 2463 & 1844 & 1275 & 2088 & \multirow{2}{*}{0.63} \\
\hline & & LD MIC & 1151 & - & 1883 & 1438 & 944 & 1712 & \\
\hline & \multirow{2}{*}{ S. salford } & DD MIC & 1056 & 3055 & 884 & 953 & 766 & 826 & \multirow{2}{*}{0.31} \\
\hline & & LD MIC & 676 & 2627 & 628 & 1077 & 537 & 576 & \\
\hline & \multirow{2}{*}{ S. newport } & DD MIC & 2280 & 2865 & 1650 & 1822 & 362 & 754 & \multirow{2}{*}{0.63} \\
\hline & & LD MIC & 2029 & 2235 & 1188 & 1330 & 260 & 498 & \\
\hline & \multirow{2}{*}{ S. sonnei } & DD MIC & 1263 & 2528 & 1050 & 1264 & 408 & 983 & \multirow{2}{*}{0.31} \\
\hline & & LD MIC & 973 & 1770 & 704 & 860 & 258 & 600 & \\
\hline & \multirow{2}{*}{ Y. enterocolitica } & DD MIC & 1358 & - & 1142 & 1679 & 985 & 1033 & \multirow{2}{*}{0.63} \\
\hline & & LD MIC & 1032 & - & 834 & 1847 & 713 & 846 & \\
\hline \multirow{12}{*}{ Gram-positive bacteria } & \multirow{2}{*}{ B. cereus } & DD MIC & 1287 & - & 852 & - & 710 & 1055 & \multirow{2}{*}{0.31} \\
\hline & & LD MIC & 940 & - & 699 & - & 440 & 1013 & \\
\hline & \multirow{2}{*}{ B. subtilis } & DD MIC & 1532 & - & 1146 & - & 1050 & 942 & \multirow{2}{*}{0.63} \\
\hline & & LD MIC & 1551 & - & 1042 & - & 872 & 688 & \\
\hline & \multirow{2}{*}{ C. perfringens } & DD MIC & 1033 & - & 1462 & - & 785 & 1104 & 063 \\
\hline & & LD MIC & 806 & - & 1257 & - & 527 & 751 & 0.03 \\
\hline & & DD MIC & 885 & 1066 & 750 & 1426 & 435 & 688 & \\
\hline & S. aureus & LD MIC & 788 & 906 & 510 & 1255 & 296 & 482 & 0.31 \\
\hline & & DD MIC & 573 & 927 & 776 & 1232 & 326 & 506 & \\
\hline & dis & LD MIC & 378 & 853 & 636 & 1060 & 231 & 349 & 0.31 \\
\hline & & DD MIC & 826 & 1850 & 983 & 1363 & 785 & 887 & \\
\hline & S. pyogenes & LD MIC & 686 & 1817 & 826 & 1063 & 573 & 585 & 0.63 \\
\hline & Giardia duoder & alis $\mathrm{IC}_{50}$ & WND & WND & WND & WND & WND & WND & 22 \\
\hline Antıprotozoal and antiviral activity & MS2 phage & IC50 & 2238 & WND & 1140 & WND & 427 & 2950 & 595 \\
\hline Anticancer activity & Caco2 cells & $\mathrm{IC}_{50}$ & - & WND & WND & WND & 885 & WND & 65 \\
\hline Anticancer activity & HeLa cells & $\mathrm{IC}_{50}$ & - & 450 & 588 & WND & 85 & 126 & 82 \\
\hline Toxicity & $\begin{array}{r}\text { Artemia francisc } \\
24 \mathrm{~h} \mathrm{LO}\end{array}$ & na nauplii & NA & NA & 1654 & NA & NA & NA & 126 \\
\hline & HDF toxi & city & NT & NT & NT & NT & NT & NT & - \\
\hline
\end{tabular}

Numbers indicate the mean $\mathrm{IC}_{50}$ or $\mathrm{LC}_{50}$ values of three experiments performed in triplicate $(n=9)$. DD MIC $=$ minimum inhibitory concentration determined by the disc diffusion assay; LD MIC = minimum inhibitory concentration determined by the liquid dilution assay; - indicates no significant growth inhibition/brine shrimp mortality; NA indicates that the extract was not significantly different from the negative control at any concentration tested; therefore, an $\mathrm{IC}_{50}$ value was not able to be determined; WND indicates $\mathrm{IC}_{50}$ was not determined as the amount of live Giardia did not reach $\leq 50 \%$ at any concentration tested; $\mathrm{NT}=$ not toxic; $\mathrm{BO}=B$. obovata decoction; $\mathrm{CE}=C$. equisetifolia decoction; $\mathrm{ET}=E$. tetrodonta decoction; $\mathrm{PC}=P$. careya decoction; $\mathrm{TC}=T$. carpentariae decoction; $\mathrm{VV}=V$. vexillata extract. ${ }^{*}$ Positive controls used for the antibacterial, Giardia, MS2 phage, cancer proliferation, and toxicity assays were gentamicin, metronidazole, C. sinensis, cisplatin, and potassium dichromate, respectively.

was tested across a range of concentrations. The C. equisetifolia, E. tetrodonta, and V. vexillata decoctions were also strong inhibitors of HeLa cell growth, inhibiting proliferation by $60-90 \%$ (Figure $6(\mathrm{~b})$ ). Furthermore, $\mathrm{IC}_{50}$ values against HeLa of 450,588 , and $126 \mu \mathrm{g} / \mathrm{mL}$ were calculated for these decoctions, respectively.

3.6. Quantification of Toxicity. All extracts were initially screened at $1000 \mu \mathrm{g} / \mathrm{mL}$ in the Artemia nauplii lethality bioassay as $\mathrm{LC}_{50}$ values $>1000 \mu \mathrm{g} / \mathrm{mL}$ have previously been defined as nontoxic [33]. Potassium dichromate was also included in the bioassay at $1000 \mu \mathrm{g} / \mathrm{mL}$ as a positive control. Potassium dichromate was rapid in its induction of mortality, with significant mortality noted by 4 hours of exposure (unpublished results). The decoctions were substantially slower at inducing mortality, with $\geq 12$ hours needed for mortality induction to become evident. Furthermore, except for the E. tetrodonta decoction, the traditional medicines did not induce Artemia nauplii mortality significantly different to that of the artificial seawater control following $24 \mathrm{~h}$ exposure (Figure 7 ). In contrast, substantial mortality was evident when nauplii were exposed to the E. tetrodonta decoction. As $\mathrm{LC}_{50}$ values $>1000 \mu \mathrm{g} / \mathrm{mL}$ have previously been defined as being nontoxic [34], only the E. tetrodonta decoction displayed apparent toxicity. Interestingly, the observed mortality substantially increased compared to that of the negative control for all decoctions 


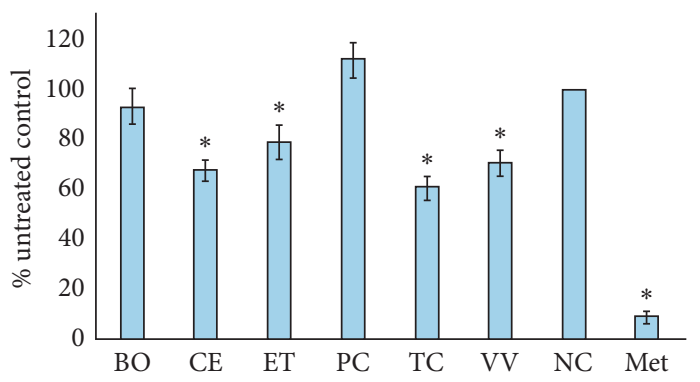

(a)

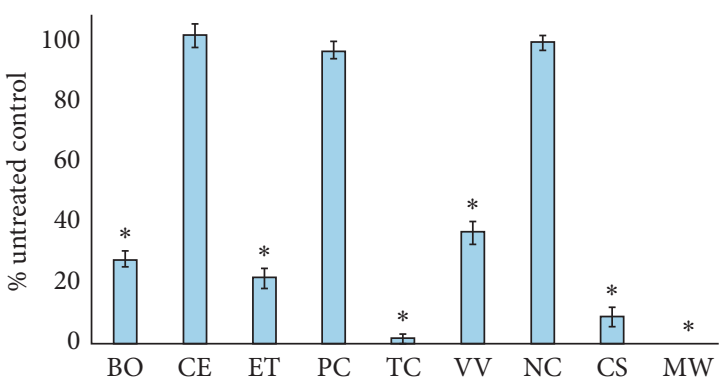

(b)

FIGURE 5: Inhibitory activity of decoctions against (a) Giardia duodenalis trophozoites and (b) MS2 bacteriophage measured as a percentage of the untreated control. $\mathrm{BO}=B$. obovata decoction; $\mathrm{CE}=C$. equisetifolia decoction; $\mathrm{ET}=$ E. tetrodonta decoction; $\mathrm{PC}=P$. careya decoction; $\mathrm{TC}=T$. carpentariae decoction; $\mathrm{VV}=V$. vexillata extract; $\mathrm{NC}=$ negative control (seawater); Met $=$ metronidazole, $\mathrm{CS}=C$. sinensis control; $\mathrm{MW}=$ microwave control. Results are expressed as mean \pm SEM of three independent experiments in triplicate $(n=9) .{ }^{*}$ indicates results that are significantly different to the untreated control $(P<0.01)$.

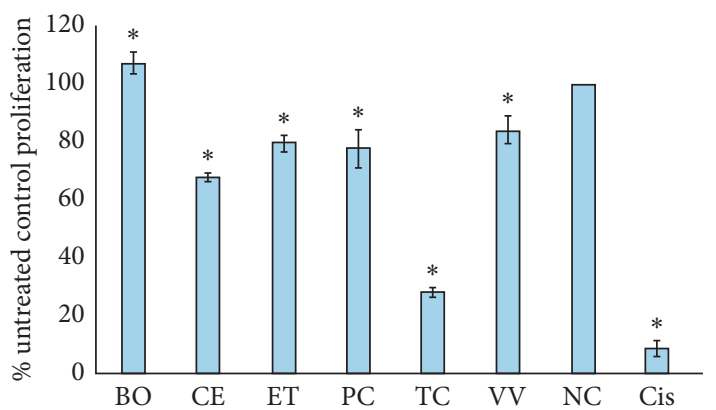

(a)

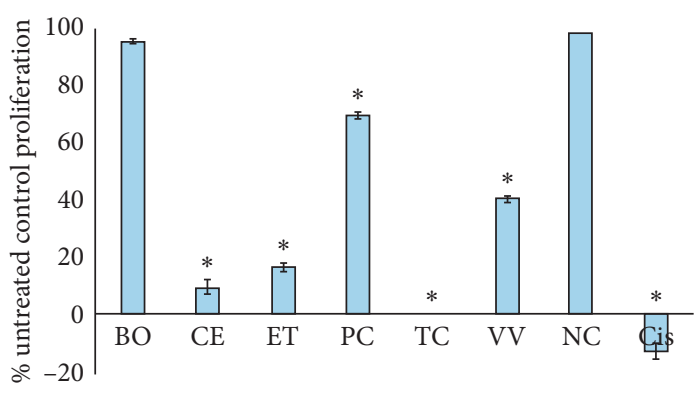

(b)

Figure 6: Antiproliferative activity of decoctions and controls against (a) Caco2 and (b) HeLa cancer cell lines measured as percentages of the untreated control cells. $\mathrm{BO}=B$. obovata decoction; $\mathrm{CE}=C$. equisetifolia decoction; $\mathrm{ET}=E$. tetrodonta; $\mathrm{PC}=P$. careya decoction; $\mathrm{TC}=T$. carpentariae decoction; $\mathrm{VV}=V$. vexillata decoction; $\mathrm{NC}=$ negative control (seawater); Cis = cisplatin control. Results are expressed as mean \pm SEM of three independent experiments in triplicate $(n=9) .{ }^{*}$ indicates results that are significantly different to the untreated control $(P<0.01)$.

except the $V$. vexillata decoction following $48 \mathrm{~h}$ exposure. As only the E. tetrodonta decoction induced significant mortality at $24 \mathrm{~h}$, only this extract was further evaluated across a range of concentrations, and $\mathrm{LC}_{50}$ was determined (Table 2 ). Notably, $\mathrm{LC}_{50}$ of $1654 \mu \mathrm{g} / \mathrm{mL}$ was determined for this decoction, and it was therefore also deemed to be nontoxic. All decoctions were also screened for toxicity in an HDF cell viability assay. Exposure of the HDF to all decoctions resulted in $>50 \%$ cell viability at $200 \mu \mathrm{g} / \mathrm{mL}$, thus confirming that all decoctions were nontoxic.

\section{Discussion}

Recent increases in the rates of microbial resistance to clinically used antibiotics have rendered many frontline treatments to be ineffective against pathogenic diseases. This is particularly true for diarrhoea-causing pathogens. The gastrointestinal system is an ideal environment not only for microbial growth but also for the exchange of genetic information between different microbial strains, and even between different species. When a pathogen in this environment possesses antibiotic resistance genes, it can readily exchange those genes with other microbes, and individual pathogens can accumulate resistance to multiple conventional antibiotics. There is an urgent need to develop new antibiotic therapies to treat diseases caused by these pathogens. For reasons reviewed elsewhere [3], the previous methods of antibiotic discovery are unlikely to yield many new antibiotics in the future, and medical science must explore new methods to treat pathogenic diseases. A reexamination of traditional medicine is an attractive option as many traditional medicines have been used effectively for hundreds or even thousands of years. Furthermore, this use has often been well documented, allowing for selection of traditional therapies for screening. Indeed, there has been a significant increase in published studies into traditional herbal therapies to treat pathogenic diseases in most regions of the world. In contrast, the traditional remedies used by the first Australians have been relatively neglected. This may be due to a less extensive reporting of Australian Aboriginal traditional remedies, which hampers studies based on directed species selection. However, the use of plant-based medicines by some groups of first Australians, including the tribes inhabiting Groote 


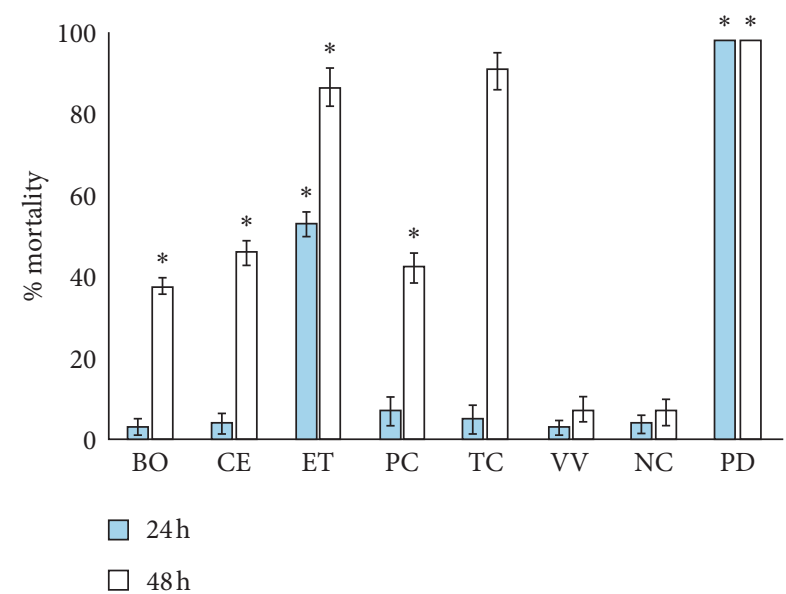

Figure 7: The lethality of the decoctions $(1000 \mu \mathrm{g} / \mathrm{mL})$ and potassium dichromate control $(1000 \mu \mathrm{g} / \mathrm{mL})$ towards Artemia franciscana nauplii after 24- or 48-hour exposure. $\mathrm{BO}=B$. obovata decoction; $\mathrm{CE}=C$. equisetifolia decoction; $\mathrm{ET}=E . \quad$ tetrodonta decoction; $\mathrm{PC}=P$. careya decoction; $\mathrm{TC}=T$. carpentariae decoction; $\mathrm{VV}=V$. vexillata decoction; $\mathrm{NC}=$ negative control (seawater); $\mathrm{PD}=$ positive control (potassium dichromate). Results are expressed as mean \pm SEM of at least triplicate determinations. ${ }^{*}$ indicates results that are significantly different to the untreated control $(P<0.01)$.

Eylandt, has been more extensively reported, allowing for more directed studies.

This study chose plant species that were recorded as being used traditionally by the Warnindhilyagwa tribe on Groote Eylandt to treat diarrhoea and other gastrointestinal complaints. The list of identified plants was further focused following surveys and consultations with Warnindhilyagwa tribal elders. As this study aimed to validate the antipathogenic activity of the traditional Warnindhilyagwa therapies, we used decoctions prepared by the tribal elders by traditional methods and tested them undiluted, as they were used traditionally. The decoctions were initially screened against a panel of bacterial pathogens selected as they are all associated with diarrhoea and gastrointestinal disease. Escherichia coli is a common trigger of diarrhoea, particularly in children [35]. Staphylococcus spp. (including S. aureus and S. epidermidis) are common causes of antibiotic-associated nosocomial diarrhoea [36]. Bacillus spp. (including B. cereus and B. subtilis) and Clostridium spp. (including C. perfringens) release diarrheagenic toxins in food poisoning cases [37]. Similarly, Shigella sonnei can cause shigellosis [38]. A recent outbreak of fatal diarrhoea was attributed to Aeromonas hydrophila [39], whilst other food-borne strains including Salmonella spp., A. faecalis, and Y. enterocolitica [40] inhabit the lower gut and cause acute diarrhoea.

Most of the decoctions were effective at inhibiting microbial growth at low concentrations, with liquid dilution MIC values against the bacterial species that they inhibited generally substantially $<1000 \mu \mathrm{g} / \mathrm{mL}$, indicating the noteworthy antimicrobial activity of these preparations. Interestingly, the tested decoctions were generally effective against both Gram-positive and Gram-negative bacteria, with similar efficacies. The ability of plant extracts to inhibit the growth of both Gram-positive and Gram negativebacteria has been previously reported for other plants that have a history of medicinal usage for the treatment of microbial diseases. The antiseptic properties of the Eucalyptus spp. [41], Leptospermum spp. [42], and Syzygium spp. [43-47] have been studied extensively and shown to inhibit the growth of a wide variety of bacteria. However, the equal or greater susceptibility of the Gram-negative bacterial species towards the plant extracts is noteworthy. This is in contrast to other previous studies which have reported a greater susceptibility of Gram-positive bacteria towards solvent extracts for South American [6], African [48], and Australian plant extracts $[43,49]$, although other examples of Australian plant extracts which have a greater effects on Gram-negative bacteria have also been reported $[22,33]$. The $T$. carpentariae and $V$. vexillata decoctions were particularly good antibacterial agents, with MIC values generally $<1000 \mu \mathrm{g} / \mathrm{mL}$ against most of the tested bacterial pathogens.

Whilst the MIC values reported in our study are noteworthy and validate the use of these plants for the treatment of bacterial diarrhoea, substantially higher MIC values are reported compared to several other Australian native plants. In particular, Terminalia ferdinandiana Excell. fruit and leaf extracts have been reported to have substantially lower MIC values against some gastrointestinal and diarrhoea-causing bacterial pathogens, with MIC values as low as $30 \mu \mathrm{g} / \mathrm{mL}$ against some species [14, 15]. Furthermore, the T. ferdinandiana extracts were not only inhibitory against susceptible reference strains of $E$. coli and $S$. aureus but also had similar potency against extended spectrum beta-lactamase and methicillin-resistant strains, indicating that the extracts may function by distinct pathways, allowing them to bypass the bacterial resistance mechanisms [15]. That study identified a diversity and relative abundance of tannins, flavonoids, and terpenoids in the extracts and deduced that they contribute to their antibacterial activity. The phytochemical constituents of the decoctions tested in our study are yet to be evaluated, and future studies are planned to assess this. Furthermore, further work is required to determine whether the decoctions tested in our study are also inhibitory towards resistant bacterial pathogens.

Most previous studies screening Australian plant extracts for potential in treating gastrointestinal and diarrhoeal diseases have myopically focused on only a few common bacterial pathogens and neglected pathogens such as C. perfringens and Y. enterocolitica. However, these bacteria also cause a substantial incidence of gastrointestinal disease and should not be neglected. Notably, several of the decoctions studied herein had noteworthy activity against these bacteria. Similarly, most previous studies have also neglected testing against nonbacterial 
pathogens. The gastrointestinal protozoal pathogen G. duodenalis is particularly common and causes substantial illness globally. Indeed, approximately 280 million people per year were reported with giardiasis internationally in a 2013 study $[49,50]$. All the decoctions tested in our study (except $B$. obovata and $P$. careya) significantly inhibited $G$. duodenalis proliferation when tested undiluted. However, none of the decoctions were particularly strong inhibitors of $G$. duodenalis proliferation, and we were unable to determine $\mathrm{IC}_{50}$ values for any extract as none inhibited proliferation by $>50 \%$ at any concentration tested. This is in contrast to several other Australian plants for which good anti-Giardial activity has been reported. Notably, extracts produced from the fruit of T. ferdinandiana are strong inhibitors of G. duodenalis growth, with $\mathrm{IC}_{50}$ values as low as $50 \mu \mathrm{g} / \mathrm{mL}$ [16]. It is therefore surprising that only low activity was noted for the $T$. carpentariae extract tested in this study. Notably, the previous study determined that the anti-Giardial activity of $T$. ferdinandiana corresponded to extracts rich in tannins, which are common to many Terminalia spp., including $T$. carpentariae. Subsequent studies have reported that the anti-Giardial activity of individual T. ferdinandiana components was potentiated by other extract components [18]. Thus, it is possible that even if the inhibitory components were present in the extract screened in this study, they may have only displayed weak activity if the potentiating components were absent. Of further note, fruit extracts were screened in the previous T. ferdinandiana studies, whereas cambium extracts were tested in this study. It is likely that the different plant parts may have substantial different phytochemical profiles, and this may account for the activity differences.

Several of the decoctions tested in this study displayed noteworthy inhibition of MS2 phage replication, with the $T$. carpentariae decoction being the most potent $\left(\mathrm{IC}_{50}=427 \mu \mathrm{g} / \mathrm{mL}\right)$. This may indicate that this decoction would be particularly useful in treating gastrointestinal diseases caused by viral pathogens such as Norovirus. These results compare favourably to other studies, which have reported antiviral activity using MS2 assays. An earlier study from our group reported comparable inhibitory activity for Scaevola spinescens R.Br. against the same virus and classified it as strong antiviral activity [33]. Notably, that study used Camellia sinensis (L.) Kuntze as the positive control and reported a substantially lower anti-MS2 phage activity $\left(\mathrm{IC}_{50}>600 \mu \mathrm{g} / \mathrm{mL}\right)$. Our study chose to screen the decoctions against the MS2 bacteriophage as a model for viral pathogens. Whilst this virus has previously been reported to be a good model to indicate activity against RNA viruses [28], we did not screen the decoctions against specific human viral pathogens, and further work is required to confirm the potential of these decoctions in treating viral gastrointestinal pathogens.

Antiproliferative activity against $\mathrm{Caco} 2$ (colorectal) and HeLa (cervical) carcinoma cell line cells was also noted for several of the decoctions, although the T. carpentariae decoction was the most potent inhibitor of proliferation against both cell lines, with $\mathrm{IC}_{50}$ values generally approximately 885 and $85 \mu \mathrm{g} / \mathrm{mL}$ against Caco 2 and HeLa cells, respectively. The antiproliferative activity of the $T$. carpentariae decoction was particularly noteworthy as colorectal cancers cause substantial mortality globally. Notably, anticancer activity has also been reported for related Terminalia spp. A recent study reported potent antiproliferative activity for $T$. ferdinandiana extracts, with $\mathrm{IC}_{50}$ values of approximately $100 \mu \mathrm{g} / \mathrm{mL}$ against Caco2 cells, which is nearly an order of magnitude stronger than $T$. carpentariae screened in these studies [31]. Fruit and leaf extracts were tested in the T. ferdinandiana study, whereas cambium decoctions were tested in this study, which may account for these broad differences. Interestingly, the $T$. ferdinandiana study determined that the extracts induced their anticancer activity by elevating caspase- 3 activity and inducing apoptosis. It is yet to be determined whether the same mechanisms are involved in the antiproliferative activity reported for the $T$. carpentariae decoction tested herein, and future studies are required to address this. Similarly, the $T$. ferdinandiana study also identified a diversity of tannins and flavonoids as contributing to the anticancer activity of those extracts [31]. Further study is required to determine if similar compounds contribute to the activity of the $T$. carpentariae extracts. The C. equisetifolia $(450 \mu \mathrm{g} / \mathrm{mL})$, E. tetrodonta $(588 \mu \mathrm{g} / \mathrm{mL})$, and $V$. vexillata $(126 \mu \mathrm{g} / \mathrm{mL})$ decoctions were also good inhibitors of HeLa proliferation but were substantially less effective against $\mathrm{Caco} 2$ cells.

Identification of the specific components responsible for the antimicrobial activity reported in the plant decoctions tested herein was beyond the scope of our study, although all decoctions were abundant in phenolics, flavonoids, and tannins. Many studies have reported potent growth inhibitory activities for a wide variety of flavonoids against extensive bacterial panels [51]. Similarly, several tannin compounds have bacterial growth inhibitory activity. Gallotannins have been reported to inhibit the growth of a broad spectrum of bacterial species [52] through a variety of mechanisms including binding cell surface molecules including lipoteichoic acid and prolinerich cell surface proteins $[53,54]$ and by inhibiting glucosyltransferase enzymes [55]. Ellagitannins are also highly potent inhibitors of bacterial growth, with MIC values as low as $63 \mu \mathrm{g} / \mathrm{mL}[52,54]$. Ellagitannins have also been reported to function via several antibiotic mechanisms including interaction with cytoplasmic oxidoreductases and by disrupting bacterial cell walls $[52,54]$. Thus, it is likely that multiple compounds within the tested decoctions contribute to the antimicrobial properties of these extracts.

The findings reported here also show that none of the decoctions displayed significant toxicity towards A. franciscana or HDFs. Whilst this indicates that these decoctions are safe to use therapeutically, further toxicity studies using other human cell lines are needed to further evaluate the suitability of the decoctions for medicinal purposes. The results of this study indicate that all the decoctions screened in this report are worthy of further study 
due to their antipathogenic activities and their abilities to block cancer cell proliferation.

\section{Conclusions}

The results of this study partially validate the traditional usage of several native Groote Eylandt plant decoctions to treat pathogenic diarrhoeal diseases and cancer, indicating that they warrant further study. The potential of T. carpentariae in the treatment of bacterial diarrhoea was particularly evident, with MIC values $230-350 \mu \mathrm{g} / \mathrm{mL}$ recorded for C. freundii, S. newport, S. sonnei, S. aureus, and S. epidermidis. Notably, T. carpentariae also displayed noteworthy inhibitory activity (MICs 450-950 $\mu \mathrm{g} / \mathrm{mL}$ ) against all other bacterial pathogens. B. obovata Engl. and E. tetrodonta were also good inhibitors of bacterial growth, albeit with substantially higher MIC values than determined for $T$. carpentariae. The T. carpentariae decoction was also the best inhibitor of MS2 phage replication $\left(\mathrm{IC}_{50}=427 \mu \mathrm{g} /\right.$ $\mathrm{mL}$ ) and $\mathrm{Caco} 2$ and HeLa proliferation $\left(\mathrm{IC}_{50}\right.$ values of 885 and $85 \mu \mathrm{g} / \mathrm{mL}$, respectively). None of the extracts were particularly strong inhibitors of Giardia duodenalis growth, indicating that none of these plants would be useful against giardiasis. From these results, T. carpentariae stands out as the most promising species for further study. Bioactivitydriven purifications of the active components and an examination of the mechanisms of action of these agents are required.

\section{Data Availability}

All data are either presented in this study or are available from the corresponding author upon request.

\section{Conflicts of Interest}

The authors declare no conflicts of interest.

\section{Acknowledgments}

The authors are grateful to Gayangwa Lalara and Gwen Lalara and other members of the Warnindhilyagwa tribe of Groote Eylandt for identifying and providing the plant material used in this study. Furthermore, they are grateful to them for producing the decoctions by traditional methods that were screened herein. They also thank David Boehme of Northern Territory Wild Harvest for facilitating Dr. Cock's visit to Groote Eylandt and arranging a meeting with the Warnindhilyagwa tribal elders. They are also grateful to Ann McDonnell for providing G. duodenalis used in these studies. Financial support for this work was provided by the Environmental Futures Research Institute and the School of Environment and Science, Griffith University.

\section{References}

[1] World Health Organization, Diarrhoea: Why Children Are Still Dying and What Can Be Done, WHO, Geneva, Switzerland, 2009, http://apps.who.int/iris/bitstream/10665/ 44174/1/9789241598415_eng.pdf.
[2] Unicef, http://data.unicef.org/child-health/diarrhoeal-disease. html, 2016.

[3] M. J. Cheesman, A. Ilanko, B. Blonk, and I. E. Cock, "Developing new antimicrobial therapies: are synergistic combinations of plant extracts/compounds with conventional antibiotics the solution?" Pharmacognosy Reviews, vol. 11, no. 22, pp. 57-72, 2017.

[4] R. Lauche, S. Kumar, J. Hallmann et al., "Efficacy and safety of ayurvedic herbs in diarrhoea-predominant irritable bowel syndrome: a randomised controlled crossover trial," Complementary Therapies in Medicine, vol. 26, pp. 171-177, 2016.

[5] A. Mishra, A. Seth, and S. Maurya, "Therapeutic significance and pharmacological activities of antidiarrhoeal medicinal plants mention in ayurveda: a review," Journal of Intercultural Ethnopharmacology, vol. 5, no. 3, p. 290, 2016.

[6] Y. S. Biradar, R. Singh, and K. Sharma, "Evaluation of antidiarrhoeal property and acute toxicity of Triphala Mashi, an ayurvedic formulation," Journal of Herbal Pharmacotherapy, vol. 7, no. 3-4, pp. 203-213, 2008.

[7] Y. Gao, H. Li, H. Yang, J. Su, and L. Huang, "The current novel therapeutic regimens for Clostridium difficile infection (CDI) and the potentials of traditional Chinese medicine in treatment of CDI," Critical Reviews in Microbiology, vol. 45, no. 56, pp. 729-742, 2019.

[8] M. Chen, T.-C. Tang, Y. Wang et al., "Randomised clinical trial: Tong-Xie-Yao-Fang granules versus placebo for patients with diarrhoea-predominant irritable bowel syndrome," Alimentary Pharmacology \& Therapeutics, vol. 48, no. 2, pp. 160-168, 2018.

[9] D. Levitt, Plants and People: Aboriginal Uses of Plants on Groote Eylandt, Australian Institute on Aboriginal Studies, Australia, 1981.

[10] E. A. Palombo and S. J. Semple, "Antibacterial activity of traditional Australian medicinal plants," Journal of Ethnopharmacology, vol. 77, no. 2-3, pp. 151-157, 2001.

[11] C. Kurekci, S. L. Bishop-Hurley, P. E. Vercoe, Z. Durmic, R. A. M. Al Jassim, and C. S. McSweeney, "Screening of Australian plants for antimicrobial activity against Campylobacter jejuni," Phytotherapy Research, vol. 26, no. 2, pp. 186-190, 2012.

[12] C. Hart, P. Ilanko, J. Sirdaarta, P. Rayan, P. A. McDonnell, and I. E. Cock, "Tasmannia stipitata as a functional food/natural preservative: antimicrobial activity and toxicity," Pharmacognosy Communications, vol. 4, no. 4, pp. 33-47, 2014.

[13] V. Winnett, H. Boyer, J. Sirdaarta, and I. E. Cock, "The potential of Tasmannia lanceolata as a natural preservative and medicinal agent: antimicrobial activity and toxicity," Pharmacognosy Communication, vol. 4, no. 1, pp. 42-52, 2014.

[14] I. E. Cock and S. Mohanty, "Evaluation of the antibacterial activity and toxicity of Terminalia ferdinandia fruit extracts," Pharmacognosy Journal, vol. 3, no. 20, pp. 72-79, 2011.

[15] M. J. Cheesman, A. White, B. Matthews, and I. E. Cock, "Terminalia ferdinandiana fruit and leaf extracts inhibit methicillin-resistant Staphylococcus aureus growth," Planta Medica, vol. 85, no. 16, p. 1253, 2019.

[16] P. Rayan, B. Matthews, P. A. McDonnell, and I. E. Cock, "Terminalia ferdinandiana extracts as inhibitors of Giardia duodenalis proliferation: a new treatment for giardiasis," Parasitology Research, vol. 114, no. 7, pp. 2611-2620, 2015.

[17] P. Rayan, B. Matthews, P. Edwin Cock, and I. E. Cock, "Phytochemical analysis of Tasmannia lanceolata extracts and inhibition of Giardia duodenalis proliferation," Pharmacognosy Journal, vol. 8, no. 3, pp. 291-299, 2016. 
[18] P. Rayan and I. E. Cock, "Ascorbic acid potentiates the Giardia duodenalis growth inhibitory activity of pure Terminalia ferdinandiana Exell compounds," Parasitology Research, vol. 119, pp. 1125-1137, 2020.

[19] C. Mazerand and I. E. Cock, "An examination of the antibacterial, antifungal, anti-giardial and anticancer properties of buchanania obovata Engl. fruit extracts," Pharmacognosy Communications, vol. 9, no. 1, pp. 7-14, 2019.

[20] K. Abba, R. Sinfield, C. A. Hart, and P. Garner, "Pathogens associated with persistent diarrhoea in children in low and middle income countries: systematic review," BMC Infectious Diseases, vol. 9, no. 8, p. 21, 2009.

[21] International Society of Ethnobiology, International Society of Ethnobiology Code of Ethics, 2008, http://ethnobiology. net/code-of-ethics/.

[22] J. Vesoul and I. Cock, "An examination of the medicinal potential of Pittosporum phylliraeoides: toxicity, antibacterial and antifungal activities," Pharmacognosy Communications, vol. 1, no. 2, pp. 8-17, 2011.

[23] F. R. Kalt and I. E. Cock, "GC-MS analysis of bioactive Petalostigma extracts: toxicity, antibacterial and antiviral activities," Pharmacognosy Magazine, vol. 10, no. 1 Suppl, pp. S37-S48, 2014.

[24] V. Winnett, J. Sirdaarta, A. White, F. M. Clarke, and I. E. Cock, "Inhibition of Klebsiella pneumoniae growth by selected Australian plants: natural approaches for the prevention and management of ankylosing spondylitis," Inflammopharmacology, vol. 25, no. 2, pp. 223-235, 2017.

[25] C. Rabadeaux, L. Vallette, J. Sirdaarta, C. Davis, and I. E. Cock, "An examination of the antimicrobial and anticancer properties of khaya senegalensis (desr.) A. Juss. Bark extracts," Pharmacognosy Journal, vol. 9, no. 4, pp. 504-518, 2017.

[26] A. Ilanko and I. E. Cock, "The interactive antimicrobial activity of conventional antibiotics and Petalostigma spp. extracts against bacterial triggers of some autoimmune inflammatory diseases," Pharmacognosy Journal, vol. 11, no. 2, pp. 292-309, 2019.

[27] A. Hutchings and I. E. Cock, "An interactive antimicrobial activity of Embelica officinalis gaertn. fruit extracts and conventional antibiotics against some bacterial triggers of autoimmune inflammatory diseases," Pharmacognosy Journal, vol. 10, no. 4, pp. 654-662, 2018.

[28] I. Cock and F. Kalt, "A modified MS2 bacteriophage plaque reduction assay for the rapid screening of antiviral plant extracts," Pharmacognosy Research, vol. 2, no. 4, pp. 221-228, 2010.

[29] I. E. Cock and F. R. Kalt, "GC-MS analysis of a Xanthorrhoea johnsonii leaf extract displaying apparent anaesthetic effects," Journal of Natural Pharmaceuticals, vol. 3, no. 2, pp. 77-88, 2012.

[30] I. E. Cock and D. R. Ruebhart, "Comparison of the brine shrimp nauplii bioassay and the ToxScreen-II test for the detection of toxicity associated with Aloe vera (Aloe barbadensis Miller) leaf extract," Pharmacognosy Journal, vol. 1, no. 2, pp. 98-101, 2009.

[31] J. Shalom and I. E. Cock, "Terminalia ferdinandianaExell. fruit and leaf extracts inhibit proliferation and induce apoptosis in selected human cancer cell lines," Nutrition and Cancer, vol. 70, no. 4, pp. 579-593, 2018.

[32] N. Jamieson, J. Sirdaarta, and I. E. Cock, "The anti-proliferative properties of Australian plants with high antioxidant capacities against cancer cell lines," Pharmacognosy Communications, vol. 4, no. 4, pp. 71-82, 2014.
[33] I. Cock and L. Kukkonen, "An examination of the medicinal potential of Scaevola spinescens: toxicity, antibacterial, and antiviral activities," Pharmacognosy Research, vol. 3, no. 2, pp. 85-94, 2011.

[34] J. Sirdaarta and I. E. Cock, "Vitamin E and Trolox ${ }^{\mathrm{TM}}$ reduce toxicity of Aloe barbadensis Miller juice in Artemia franciscana nauplii but individually are toxic at high concentrations," Internet Journal of Toxicology, vol. 5, no. 1, 2008.

[35] L. Cabrera-Sosa and T. J. Ochoa, "Escherichia coli diarrhea," in Hunter's Tropical Medicine and Emerging Infectious Diseases, pp. 481-485, Elsevier, Amsterdam, Netherlands, 2020.

[36] C. R. Polage, J. V. Solnick, and S. H. Cohen, "Nosocomial diarrhea: evaluation and treatment of causes other than Clostridium difficile," Clinical Infectious Diseases, vol. 55, no. 7, pp. 982-989, 2012.

[37] A. K. Bhunia, Foodborne Microbial Pathogens: Mechanisms and Pathogenesis, Springer, Berlin, Germany, 2018.

[38] K. L. Kotloff, M. S. Riddle, J. A. Platts-Mills, P. Pavlinac, and A. K. M. Zaidi, "Shigellosis," The Lancet, vol. 391, no. 10122, pp. 801-812, 2018.

[39] J. Liu, L. Xie, D. Zhao et al., "A fatal diarrhoea outbreak in farm-raised Deinagkistrodon acutus in China is newly linked to potentially zoonotic Aeromonas hydrophila," Transboundary and Emerging Diseases, vol. 66, no. 1, pp. 287-298, 2019.

[40] S. Y. Wotzka, M. Kreuzer, L. Maier et al., "Escherichia coli limits Salmonella Typhimurium infections after diet shifts and fat-mediated microbiota perturbation in mice," Nature Microbiology, vol. 4, no. 12, pp. 2164-2174, 2019.

[41] I. E. Cock, "Antimicrobial activity of Eucalyptus major and Eucalyptus baileyana methanolic extracts," Internet Journal of Microbiology, vol. 6, no. 1, 2009.

[42] I. E. Cock, "Antimicrobial activity of Leptospermum bracteata and Letptospermum juniperium methanolic extracts," Pharmacognosy Communications, vol. 3, no. 3, pp. 45-52, 2013.

[43] C. Sautron and I. E. Cock, "Antimicrobial activity and toxicity of Syzygium australe and Syzygium leuhmanii fruit extracts," Pharmacognosy Communications, vol. 4, no. 1, pp. 53-60, 2014.

[44] G. Chikowe, L. Mpala, and I. E. Cock, "Antibacterial activity of selected Australian Syzygium species," Pharmacognosy Communications, vol. 3, no. 4, pp. 77-83, 2013.

[45] S. Mohanty and I. E. Cock, "Bioactivity of Syzygium jambos methanolic extracts: antibacterial activity and toxicity," Pharmacognosy Research, vol. 2, pp. 4-9, 2010.

[46] E. A. Paz, M. P. Cerdeiras, J. Fernandez et al., "Screening of Uruguayan medicinal plants for antimicrobial activity," Journal of Ethnopharmacology, vol. 45, no. 1, pp. 67-70, 1995.

[47] S. Murhekar, M. H. Wright, A. C. Greene, J. C. Brownlie, and I. E. Cock, "Inhibition of Shewanella spp. growth by Syzygium australe and Syzygium luehmannii extracts: natural methods for the prevention of fish spoilage," Journal of Food Science and Technology, vol. 54, no. 10, pp. 3314-3326, 2017.

[48] A. J. Vlietinck, L. van Hoof, J. Totté et al., "Screening of hundred Rwandese medicinal plants for antimicrobial and antiviral properties," Journal of Ethnopharmacology, vol. 46, no. 1, pp. 31-47, 1995.

[49] I. E. Cock, "Antibacterial activity of selected Australian plant species," The Internet Journal of Microbiology, vol. 6, no. 2, 2008.

[50] K. J. Esch and C. A. Petersen, "Transmission and epidemiology of zoonotic protozoal diseases of companion animals," Clinical Microbiology Reviews, vol. 26, no. 1, pp. 58-85, 2013. 
[51] K. R. Narayana, M. S. Reddy, M. R. Chaluvadi et al., "Bioflavonoids classification, pharmacological, biochemical effects and therapeutic potential," Indian Journal of Pharmacology, vol. 33, no. 1, pp. 2-16, 2001.

[52] P. Buzzini, P. Arapitsas, M. Goretti et al., "Antimicrobial and antiviral activity of hydrolysable tannins," Mini-Reviews in Medicinal Chemistry, vol. 8, no. 12, pp. 1179-1187, 2008.

[53] L. E. Wolinsky and E. O. Sote, "Isolation of natural plaqueinhibiting substances from "Nigerian chewing sticks"," Caries Research, vol. 18, no. 3, pp. 216-225, 1984.

[54] S. D. Hogg and G. Embery, "Blood-group-reactive glycoprotein from human saliva interacts with lipoteichoic acid on the surface of Streptococcus sanguis cells," Archives of Oral Biology, vol. 27, no. 3, pp. 261-268, 1982.

[55] C. D. Wu-Yuan, C. Y. Chen, and R. T. Wu, "Gallotannins inhibit growth, water-insoluble glucan synthesis, and aggregation of mutans streptococci," Journal of Dental Research, vol. 67, no. 1, pp. 51-55, 1988. 